\title{
Commuter of operators in a Hilbert space
}

\author{
Alain Boudou ${ }^{\mathrm{a}}$, Sylvie Viguier-Pla ${ }^{\mathrm{b}}$ \\ ${ }^{a}$ Équipe de Statistique et Probabilités, Institut de Mathématiques, UMR5219, Université Paul-Sabatier, \\ 118, route de Narbonne, F-31062 Toulouse Cedex 9, France \\ ${ }^{b}$ LAMPS, Université de Perpignan, via Domitia, 52, avenue Paul-Alduy, 66860 Perpignan Cedex 9, France
}

\begin{abstract}
Commutativity between two stationary functions is a notion that generalizes the concept of stationary correlation. Two stationary functions commute if and only if their associated spectral measures and unitary operators commute. When the operators do not commute, we define the concept of commuter for two operators, and then derive it for two projectors, for a projector and a unitary operator, and for two unitary operators. We establish relations between these commuters and some other tools related to proximity between processes. Finally, we propose a method which retrieves the commuter for two stationary series of finite spectrum, and we study some convergence properties.
\end{abstract}

Keywords: Commuter, Orthogonal projectors, Partial order relation, Random measures, Spectral measures, Stationary processes, Unitary operators.

AMS subject classification: 60G57, 60G10, 60B15, 60H05

\section{Introduction}

Methods for comparing sets of curves are largely rooted in the comparison of populations through factor analysis; see, e.g., [2, 12]. Spectral theory is well suited to the task when the curves stem from the observation of processes. This approach has been used successfully to study many issues dealing with, e.g., autoregressive processes [3], dimension reduction $[4,5]$, and large deviation theory [14]. To the best of our knowledge, however, the use of spectral elements for comparing two sets of functions has been scant. This is in spite of their obvious relevance for issues such as extracting common and specific features for two sets of curves, or retrieving all the curves with a common shape.

To avoid time dependence issues, curve comparison issues are typically addressed in the frequency domain, and harmonic analysis techniques can be used in this context. They do not rely on the Gaussian assumption commonly used in functional data analysis on functional processes; see [10] for a discussion. Dependent functional data analysis, including functional time series and spatial statistics, is treated in detail by Horváth and Kokoszka [13]. Part of these developments were stimulated by the early work of Bosq [3] for dependent data; see also Ramsay and Silverman [18].

According to Hsing and Eubank [15], functional data can be approached either from a random element perspective or from a stochastic process perspective. This paper does a bit of both. Specifically, we develop mathematical tools for stationary processes. For a stationary function, we know that there exists one and only one unitary operator, namely the shift operator. The latter can be expressed as a linear combination of projectors or more generally as an integral with respect to a projector-valued spectral measure, viz.

$$
U X=\sum_{j \in J} e^{i \lambda_{j}} P_{j} X \quad \text { or } \quad U X=\int e^{i \lambda} \mathrm{d} \mathcal{E}(\lambda) X,
$$

where $\mathcal{E}$ is a projector-valued spectral measure. A spectral measure is associated with a unitary operator $U$ in a unique way; we denote it $\mathcal{E}_{U}$. Our objective is to study the relations between the spectral measures $\mathcal{E}_{U}, \mathcal{E}_{V}, \mathcal{E}_{U V}$ and $\mathcal{E}_{V U}$, where $U$ and $V$ are unitary operators. The comparison of two random functions can then be transposed in the frequency domain through the comparison of their associated unitary operators.

Email addresses: viguier@univ-perp.fr (Sylvie Viguier-Pla), boudou@math.univ-toulouse.fr (Sylvie Viguier-Pla) 
Commutativity between two unitary operators makes complete sense in this context. When two random functions are stationarily correlated, they show some similarity. The notion of stationary correlation can be generalized by the commutativity of these functions; see [8]. In the frequency domain, this notion corresponds to the commutativity of the unitary operators, which is equivalent to the commutativity of the associated spectral measures. When two unitary operators $U$ and $V$ commute, there exists a simple relation between $\mathcal{E}_{U}, \mathcal{E}_{V}$ and $\mathcal{E}_{U V}=\mathcal{E}_{V U}$. By analogy with measure theory, it is natural to say that $\mathcal{E}_{U V}$ is the convolution product of the spectral measures $\mathcal{E}_{U}$ and $\mathcal{E}_{V}$. We emphasize that the product of convolution of spectral measures, as defined in [6], relies on a commutativity assumption. When the operators do not commute, the relation is more complex.

These considerations lead us to introduce here the notion of commuter, which retrieves the part that commutes between two operators. The study of this commuter then allows us to define how close two random functions can be. Projections play an important role in our study; for, $\mathcal{E}_{U V} A, \mathcal{E}_{V U} A$ and the commuter are projectors. We also rely on a partial order relation defined on the set of projectors [9]. Obviously, the commuter of two projectors is linked with the canonical analysis of the spaces they generate. These concepts are developed here in a general Hilbertian framework, and when the $\mathbb{C}$-Hilbert space is of the $L_{H}^{2}(\Omega, \mathcal{A}, P)$ type, our results apply to stationary processes. When the operators do not commute, we can define the convolution product on a subspace generated by the commuter. The study of unitary operators, and of their associated random functions, contributes to the development of operator-based statistical theory, with potential application to functional data analysis.

This paper is structured as follows. Prerequisites and notation are first reviewed in Section 2. The maximal commuter of two operators is then defined in Section 3, where it is discussed in three special cases: two unitary operators, a projector and a unitary operator, and two projectors. In Section 4, we examine the relations between the spectral measures associated with two unitary operators and their product. Section 5 is devoted to the study of the convergence of the case where the spectrum is finite to the continuous spectrum case. As an application, Section 6 contains a characterization of all stationary series which are stationarily correlated with a given stationary series, and which belong to a given vector subspace. A graphical illustration of the results is given in Section 7.

\section{Background and notation}

We recall here the necessary tools and fix notation. We work in the complex field to be able to use Fourier transforms. General references on stochastic integrals and the product of convolution of spectral measures are [6, 7]; see also [9] for the notions of convergence of projectors, gap between projectors, and gap between spectral measures.

In what follows, $H$ is a $\mathbb{C}$-Hilbert space and $\mathcal{L}(H)$ is the set of bounded endomorphisms, which is a $\mathbb{C}$-Banach space for the norm $\|A\|_{\mathcal{L}(H)}=\sup \{\|A X\|:\|X\|=1\}$. Let $\mathcal{P}(H)$ be the set of projectors on $H$. If $H^{\prime}$ is a closed vector subspace of $H, \mathcal{P}\left(H^{\prime}\right)$ is the set of projectors on $H^{\prime}$. Denote by $C^{\perp}$ the projector $I-C$ which is orthogonal to a projector $C$. Let $\mathcal{B}$ denote the Borel $\sigma$-field of $\Pi=[-\pi, \pi)$. For any $\lambda \in \Pi, \delta_{\lambda}$ is the Dirac measure concentrated on $\lambda$.

A random measure $Z$, defined on a $\sigma$-field $\xi$ of subsets of a set $E$ and taking values in $H$, is a vector measure such that $\langle Z A, Z B\rangle=0$ for all pairs $(A, B)$ of disjoint elements of $\xi$. It can be shown that the application $\mu_{Z}: A \in$ $\xi \mapsto\|Z A\|^{2} \in \mathbb{R}_{+}$is a bounded measure. A stochastic integral relative to the random measure $Z$ can be defined as the unique isometry from $L^{2}\left(E, \xi, \mu_{Z}\right)$ onto $H_{Z}=\overline{\operatorname{vect}}\{Z A: A \in \xi\}$ which associates $Z A$ to $\mathbf{1}_{A}$ for all $A \in \xi$. The image of an element $\varphi$ of $L^{2}\left(E, \xi, \mu_{Z}\right)$ by this isometry is called the integral of $\varphi$ with respect to the random measure $Z$ and is denoted either $\int \varphi \mathrm{d} Z$ or $\int \varphi(\lambda) \mathrm{d} Z(\lambda)$.

If $U$ is a unitary operator, then $U \circ Z$ is a random measure, $\mu_{U \circ Z}=\mu_{Z}$, and for any $\varphi \in L^{2}\left(E, \xi, \mu_{U \circ Z}\right)=L^{2}\left(E, \xi, \mu_{Z}\right)$, $\int \varphi \mathrm{d} U \circ Z=U\left(\int \varphi \mathrm{d} Z\right)$. The qualifier "random" makes sense if $H$ is of the $L^{2}(\Omega, \mathcal{A}, P)$ type.

A stationary series $\left(X_{n}, n \in \mathbb{Z}\right)$ is a family of elements of $H$ such that $\left\langle X_{n}, X_{m}\right\rangle=<X_{n-m}, X_{0}>$ for all $n, m \in \mathbb{Z}$. When $H=L^{2}(\Omega, \mathcal{A}, P)$, and when $\int X_{n} \mathrm{~d} P=0$ for every $n \in \mathbb{Z}$, we get the usual definition of stationarity of order 2 , because $<X_{n}, X_{m}>=\operatorname{cov}\left(X_{n}, X_{m}\right)$.

With any stationary series $\left(X_{n}, n \in \mathbb{Z}\right)$ of elements of $H$, we can associate a unique random measure $Z$, defined on $\mathcal{B}$ and taking values in $H$, such that $X_{n}=\int \mathrm{e}^{i \lambda n} \mathrm{~d} Z(\lambda)$ for all $n \in \mathbb{Z}$. In the following, we will also write $X_{n}=\int \mathrm{e}^{i \cdot n} \mathrm{~d} Z$, where $\mathrm{e}^{i \cdot n}$ is the application $\lambda \in \Pi \mapsto \mathrm{e}^{i \lambda n} \in \mathbb{C}$.

In the same way, we can define a stationary series indexed by $\mathbb{Z} \times \mathbb{Z}$ : a family $\left\{X_{n, m}:(n, m) \in \mathbb{Z} \times \mathbb{Z}\right\}$ of elements of $H$ is a stationary series when $\left\langle X_{n, m}, X_{n^{\prime}, m^{\prime}}\right\rangle=\left\langle X_{n-n^{\prime}, m-m^{\prime}}, X_{0,0}\right\rangle$ for all $n, n^{\prime}, m, m^{\prime} \in \mathbb{Z}$. Two stationary series $\left(X_{n}, n \in \mathbb{Z}\right)$ and $\left(Y_{n}, n \in \mathbb{Z}\right)$ are said to be stationarily correlated when $<X_{n}, Y_{m}>=<X_{n-m}, Y_{0}>$ for all $n, m \in \mathbb{Z}$. 
A spectral measure $\mathcal{E}$ on $\xi$ for $H$ is an application from $\xi$ into $\mathcal{P}(H)$ such that $\mathcal{E} E=I_{H}, \mathcal{E}(A \cup B)=\mathcal{E} A+\mathcal{E} B$ for any pair $(A, B)$ of disjoint elements of $\xi$, and $\mathcal{E} A_{n} X \rightarrow 0$ as $n \rightarrow \infty$ for any $X \in H$ and any decreasing sequence $\left(A_{n}: n \in \mathbb{N}\right)$ of elements of $\xi$ converging to $\emptyset$.

It can be shown that for any $X \in H$, the application $Z_{\mathcal{E}}^{X}: A \in \xi \mapsto \mathcal{E} A X \in H$ is a random measure. If $(F, \mathcal{F})$ is a second measurable space and $f$ is a measurable application from $E$ to $F$, then the application $f(\mathcal{E}): A \in \mathcal{F} \mapsto$ $\mathcal{E}\left(f^{-1} A\right) \in \mathcal{P}(H)$ is a spectral measure on $\mathcal{F}$ for $H$ called the spectral measure image of $\mathcal{E}$ by $f$.

With any unitary operator $U$ of $H$, we can associate a unique spectral measure on $\mathcal{B}$ for $H$, denoted $\mathcal{E}_{U}$ and called the spectral measure associated with the unitary operator $U$, such that $U X=\int \mathrm{e}^{i \lambda} \mathrm{d} Z_{\mathcal{E}_{U}}^{X}(\lambda) \in H$ for all $X \in H$. Conversely, if $\mathcal{E}$ is a spectral measure on $\mathcal{B}$ for $H$, then the application $X \in H \mapsto \int e^{i \cdot 1} \mathrm{~d} Z_{\mathcal{E}}^{X} \in H$ is a unitary operator of associated spectral measure $\mathcal{E}$. If $U$ is a unitary operator, then, for any $\lambda \in \Pi$, $\operatorname{Im} \mathcal{E}_{U}(\{\lambda\})=\left\{X \in H: U X=\mathrm{e}^{i \lambda} X\right\}$.

Let $\left\{P_{j}: j \in J\right\}$ be a finite family of projectors such that $\sum_{j \in J} P_{j}=I_{H}$ and such that $P_{j} P_{\ell}=0$ for all pairs $(j, \ell)$ of distinct elements of $J$. If $\left\{\lambda_{j}: j \in J\right\}$ is a (finite) family of distinct elements of $\Pi$, then $U=\sum_{j \in J} \mathrm{e}^{i \lambda_{j}} P_{j}$ is a unitary operator and $\mathcal{E}_{U}=\sum_{j \in J} \delta_{\lambda_{j}} P_{j}$.

For any $n \in \mathbb{Z}$, the application $\widehat{h_{n}}: \lambda \in \Pi \mapsto n \lambda-2 \pi[(n \lambda+\pi) /(2 \pi)] \in \Pi$, where $[x]$ denotes the integer part of $x$, is measurable. It can be shown that if $U$ is a unitary operator, the spectral measure associated with the unitary operator $U^{n}$ is the image by $\widehat{h_{n}}$ of the spectral measure associated with $U$. With the notational convention we have adopted, $\widehat{h_{n}}\left(\mathcal{E}_{U}\right)=\mathcal{E}_{U^{n}}$.

A bounded endomorphism $T$ of $H$ commutes with a unitary operator $U$ if and only if $\left(\mathcal{E}_{U} A\right) T=T\left(\mathcal{E}_{U} A\right)$ for all $A \in \mathcal{B}$. Thus, two unitary operators $U$ and $V$ commute if and only if, for any pair $(A, B)$ of elements of $\mathcal{B}$, the projectors $\mathcal{E}_{U} A$ and $\mathcal{E}_{V} B$ commute. We then say that the spectral measures $\mathcal{E}_{U}$ and $\mathcal{E}_{V}$ commute and it can be shown that there exists a unique spectral measure, denoted $\mathcal{E}_{U} \otimes \mathcal{E}_{V}$, on $\mathcal{B} \otimes \mathcal{B}$ for $H$, such that $\mathcal{E}_{U} \otimes \mathcal{E}_{V}(A \times B)=\left(\mathcal{E}_{U} A\right)\left(\mathcal{E}_{V} B\right)$ for all pairs $(A, B)$ of elements of $\mathcal{B}$. Note that $\left(\mathcal{E}_{U} A\right)\left(\mathcal{E}_{V} B\right)$ is a projector because $\mathcal{E}_{U} A$ and $\mathcal{E}_{V} B$ commute. The image of $\mathcal{E}_{U} \otimes \mathcal{E}_{V}$ by the measurable application $S:\left(\lambda, \lambda^{\prime}\right) \in \Pi \times \Pi \mapsto \lambda+\lambda^{\prime}-2 \pi\left[\left(\lambda+\lambda^{\prime}+\pi\right) /(2 \pi)\right] \in \Pi$, denoted $\mathcal{E}_{U} * \mathcal{E}_{V}$, is called the product of convolution of the spectral measures $\mathcal{E}_{U}$ and $\mathcal{E}_{V}$; it is associated with the unitary operator $U V$, i.e., $\mathcal{E}_{U V}=\mathcal{E}_{U} * \mathcal{E}_{V}=S\left(\mathcal{E}_{U} \otimes \mathcal{E}_{V}\right)$.

When $C$ is a projector of $H$, we denote by $L_{C}$ the application $X \in \operatorname{Im} C \mapsto X \in H$. It can be seen that $L_{C}^{*}(X)=C X$ for all $X \in H$, that $L_{C} L_{C}^{*}=C, L_{C}^{*} L_{C}=I_{\operatorname{Im} C}, L_{C}^{*} C=L_{C}^{*}$, and that $C L_{C}=L_{C}$.

A projector $C$ and a unitary operator $U$ commute if and only if $L_{C}^{*} U L_{C}$ is a unitary operator of $\operatorname{Im} C$. One can then check that for all $A \in \mathcal{B}, L_{C}^{*}\left(\mathcal{E}_{U} A\right) L_{C}$ is a projector of $\operatorname{Im} C$ and that the application $A \in \mathcal{B} \mapsto L_{C}^{*}\left(\mathcal{E}_{U} A\right) L_{C} \in \mathcal{P}(\operatorname{Im} C)$ is the spectral measure associated with the unitary operator $L_{C}^{*} U L_{C}$, i.e., $\mathcal{E}_{L_{C}^{*} U L_{C}}(A)=L_{C}^{*}\left(\mathcal{E}_{U} A\right) L_{C}$ for all $A \in \mathcal{B}$.

Finally, we recall the following notions concerning a partial order relation defined on $\mathcal{P}(H)$. Let $P$ and $Q$ be two projectors. We say that $P$ is smaller than $Q$, denoted $P \ll Q$, if one of the following equivalent properties is verified: (i) $P=P Q=Q P$; (ii) $\operatorname{Im} P \subset \operatorname{Im} Q$; (iii) $\|P X\| \leq\|Q X\|$ for all $X \in H$. Any family $\left\{P_{\lambda}: \lambda \in \Lambda\right\}$ of projectors has a greatest lower bound, denoted $\inf \left\{P_{\lambda}: \lambda \in \Lambda\right\}$, and a least upper bound, denoted $\sup \left\{P_{\lambda}: \lambda \in \Lambda\right\}$. In what follows, the following properties will often be used:

(i) $P \ll Q$ if and only if $Q^{\perp} \ll P^{\perp}$.

(ii) If $(P, Q)$ is a pair of projectors which commute, then $\inf (P, Q)=P Q$.

(iii) $\operatorname{Im} \inf \left\{P_{\lambda}: \lambda \in \Lambda\right\}=\cap_{\lambda \in \Lambda} \operatorname{Im} P_{\lambda}$.

(iv) $\left[\sup \left\{P_{\lambda}: \lambda \in \Lambda\right\}\right]^{\perp}=\inf \left\{P_{\lambda}^{\perp}: \lambda \in \Lambda\right\}$.

(v) $\left[\inf \left\{P_{\lambda}: \lambda \in \Lambda\right\}\right]^{\perp}=\sup \left\{P_{\lambda}^{\perp}: \lambda \in \Lambda\right\}$.

(vi) If a projector $C$ commutes with projectors $P$ and $Q$, then $C$ commutes with $\inf (P, Q)$, and $C \inf (P, Q)=$ $\inf (C P, C Q)$.

Let $\left(P_{n}, n \in \mathbb{N}\right)$ be a sequence of projectors. It is then possible to define its upper and lower limits as

$\limsup \left\{P_{n}: n \in \mathbb{N}\right\}=\inf \left\{\sup \left\{P_{m}: m \geq n\right\}: n \in \mathbb{N}\right\}, \quad \liminf \left\{P_{n}: n \in \mathbb{N}\right\}=\sup \left\{\inf \left\{P_{m}: m \geq n\right\}: n \in \mathbb{N}\right\}$,

respectively. We always have $\liminf \left\{P_{n}: n \in \mathbb{N}\right\} \ll \lim \sup \left\{P_{n}: n \in \mathbb{N}\right\}$. When there is equality, we say that $\left(P_{n}, n \in \mathbb{N}\right) r$-converges: $\lim _{n}^{r} P_{n}=P$ if and only if $\liminf \left\{P_{n}: n \in \mathbb{N}\right\}=\lim \sup \left\{P_{n}: n \in \mathbb{N}\right\}=P$. 
If a sequence of projections $r$-converges, then it converges point-wise, but the converse is not true. If $\left(P_{n}, n \in \mathbb{N}\right)$ is an increasing (resp. decreasing) sequence of projectors, it is $r$-convergent and $\lim _{n}^{r} P_{n}=\sup \left\{P_{n}: n \in \mathbb{N}\right\}$ (resp. $\left.\lim _{n}^{r} P_{n}=\inf \left\{P_{n}: n \in \mathbb{N}\right\}\right)$.

For any pair $(P, Q)$ of projectors, we define $d(P, Q)=\sup (P, Q)-\inf (P, Q)$. This notion looks like a distance but it is not, as $d(P, Q)$ is a projector. It is interesting nevertheless because $\lim _{n}^{r} P_{n}=P$ if and only if $\lim _{n}^{r} d\left(P_{n}, P\right)=0$. Note that $d(P, Q)=0$ if and only if $P=Q$. It can also be checked that $\operatorname{Im}\{d(P, Q)\}^{\perp}=\operatorname{Ker}(P-Q)$.

Given spectral measures $\mathcal{E}$ and $\alpha$ on $\mathcal{B}$ for $H$, their associated gap is given by $E_{\mathcal{E}, \alpha}=\sup \{d(\mathcal{E} A, \alpha A): A \in \mathcal{B}\}$. An equalizer of two unitary operators $U$ and $V$ is a projector $K$ which commutes with $U$ and $V$ and such that $U K=V K$. The upper bound of the family of the equalizers of the unitary operators $U$ and $V$ is an equalizer of $U$ and $V$, denoted $R_{U, V}$ and called the maximal equalizer of $U$ and $V$. Note that $R_{U, V}^{\perp}=E_{\mathcal{E}_{U}, \mathcal{E}_{V}}$ and $\operatorname{Im} R_{U, V}=\cap_{n \in \mathbb{Z}} \operatorname{Ker}\left(U^{n}-V^{n}\right)$.

\section{Commuters}

The notion of commuter studied here is different from the notion of commutator described in other papers, e.g., Laustsen [16], who defines the commutator of two operators $A$ and $B$ as $[A, B]=A B-B A$. We define a commuter as a projector which will be equal to the identity when the operators commute.

\subsection{Maximal commuter}

We begin by stating some preliminary results pertaining to commutativity.

Lemma 1. If the projector $P$ commutes with $A \in \mathcal{L}(H)$, then $P A^{*}=A^{*} P$ and $P^{\perp} A=A P^{\perp}$.

Proof. From $A P=P A$, we get $P A^{*}=A^{*} P$ using the adjoint. Commutativity between $P^{\perp}$ and $A$ is easily checked.

The following result is somewhat more involved.

Lemma 2. If $A \in \mathcal{L}(H)$ commutes with each projector in $\left\{P_{\lambda}: \lambda \in \Lambda\right\}$, then $M A M=A M$ with $M=\inf \left\{P_{\lambda}: \lambda \in \Lambda\right\}$.

Proof. Fix an arbitrary $X \in H$. For any $\lambda \in \Lambda$, we have $P_{\lambda} A M X=A P_{\lambda} M X=A M X$. This means that $A M X$ belongs to $\cap_{\lambda \in \Lambda} \operatorname{Im} P_{\lambda}=\operatorname{Im} \inf \left\{P_{\lambda}: \lambda \in \Lambda\right\}=\operatorname{Im} M$. So $M A M X=A M X$, which completes the proof.

The commutativity of a family of projectors implies the commutativity of the upper and the lower bounds.

Lemma 3. If $A \in \mathcal{L}(H)$ commutes with each projector in $\left\{P_{\lambda}: \lambda \in \Lambda\right\}$, then $A$ commutes with $M=\inf \left\{P_{\lambda}: \lambda \in \Lambda\right\}$ and $S=\sup \left\{P_{\lambda}: \lambda \in \Lambda\right\}$.

Proof. From Lemma 2, we have $M A M=A M$. Furthermore, from Lemma 1, $A^{*}$ commutes with each projector in $\left\{P_{\lambda}: \lambda \in \Lambda\right\}$. Lemma 2 also allows us to write $M A^{*} M=A^{*} M$, and hence $M A M=M A$. We can then conclude that $M A=A M$. Finally, from Lemma $1, A$ commutes with each projector in $\left\{P_{\lambda}^{\perp}: \lambda \in \Lambda\right\}$, i.e., with $\inf \left\{P_{\lambda}^{\perp}: \lambda \in \Lambda\right\}$, and hence with $\left[\inf \left\{P_{\lambda}^{\perp}: \lambda \in \Lambda\right\}\right]^{\perp}=\sup \left\{P_{\lambda}: \lambda \in \Lambda\right\}=S$.

We are now ready to introduce the notion of commuter.

Definition 1. A projector $K$ is a commuter of $A, B \in \mathcal{L}(H)$ if it commutes with $A$ and $B$ and $A K B=B K A$.

The upper bound of a family of commuters is a commuter.

Proposition 1. If each projector in $\left\{K_{\lambda}: \lambda \in \Lambda\right\}$ is a commuter of $A, B \in \mathcal{L}(H)$, then $K=\sup \left\{K_{\lambda}: \lambda \in \Lambda\right\}$ is a commuter of $A$ and $B$.

Proof. From Lemma 3, $A$ (resp. B) commutes with $K$. Denote by $D$ the projector on the closed vector subspace $\operatorname{Ker}(A B-B A)$. For any $\lambda \in \Lambda$, we have

$$
(A B-B A) K_{\lambda}=A B K_{\lambda}-B A K_{\lambda}=A K_{\lambda} B-B K_{\lambda} A=B K_{\lambda} A-B K_{\lambda} A=0 .
$$


This means that $\operatorname{Im} K_{\lambda} \subset \operatorname{Im} D=\operatorname{Ker}(A B-B A)$, and hence $K_{\lambda} \ll D$. The projector $D$ being an upper bound of the family $\left\{K_{\lambda}: \lambda \in \Lambda\right\}$, we have $K \ll D$. For any $X \in H$, we have $K X \in \operatorname{Im} K \subset \operatorname{Im} D=\operatorname{Ker}(A B-B A)$, so $A B K X=B A K X$. In other words, because $K$ commutes with $A$ and $B, A K B X=B K A X$. Since $X \in H$ was arbitrary, we have $A B K=B K A$. Hence the proof is complete.

As the set of the commuters of a pair $(A, B)$ of elements of $\mathcal{L}(H)$ is never empty, because 0 is a commuter of $A$ and $B$, Proposition 1 allows us to define the maximal commuter.

Definition 2. If $A$ and $B$ are elements of $\mathcal{L}(H)$, the upper bound of the family of all commuters of $A$ and $B$ is called the maximal commuter of $A$ and $B$, and it is denoted $C_{A, B}$.

In the following subsections, we consider in turn the maximal commuter of two unitary operators, of a projector and a unitary operator, and of two projectors.

\subsection{Maximal commuter of two unitary operators}

A commuter of two unitary operators can be defined by means of their associated spectral measures. For this, we establish the following preliminary result.

Lemma 4. A projector $K$ is a commuter of the unitary operators $U$ and $V$ if and only if $L_{K}^{*} U L_{K}$ and $L_{K}^{*} V L_{K}$ are unitary operators which commute.

Proof. If $K$ is a commuter of $U$ and $V, K$ commutes with $U$, so $L_{K}^{*} U L_{K}$ is a unitary operator of $\operatorname{Im} K$. For the same reasons, $L_{K}^{*} V L_{K}$ is a unitary operator of $\operatorname{Im} K$. Moreover,

$$
\left(L_{K}^{*} U L_{K}\right)\left(L_{K}^{*} V L_{K}\right)=L_{K}^{*} U K V L_{K}=L_{K}^{*} V K U L_{K}=\left(L_{K}^{*} V L_{K}\right)\left(L_{K}^{*} U L_{K}\right),
$$

so $L_{K}^{*} U L_{K}$ and $L_{K}^{*} V L_{K}$ are unitary operators of $\operatorname{Im} K$ which commute.

Conversely, if $L_{K}^{*} U L_{K}$ and $L_{K}^{*} V L_{K}$ are unitary operators of $\operatorname{Im} K$ which commute, we have that $K$ commutes with $U$ and $V$. In addition,

$$
U K V=K U K V K=L_{K} L_{K}^{*} U L_{K} L_{K}^{*} V L_{K} L_{K}^{*}=L_{K} L_{K}^{*} V L_{K} L_{K}^{*} U L_{K} L_{K}^{*}=K V K U K=V K U .
$$

Indeed, the projector $K$ is a commuter of the unitary operators $U$ and $V$.

We can now establish a relationship between the commuter of two unitary operators and the commuter of a family of pairs of projectors.

Proposition 2. A projector $K$ is a commuter of the unitary operators $U$ and $V$ if and only if, for all pairs $(A, B)$ of elements of $\mathcal{B}, K$ is a commuter of the projectors $\mathcal{E}_{U} A$ and $\mathcal{E}_{V} B$.

Proof. Let $K$ be a commuter of $U$ and $V$. As $K$ commutes with $U$ (resp. $V$ ), $L_{K}^{*} U L_{K}$ (resp. $L_{K}^{*} V L_{K}$ ) is a unitary operator of $\operatorname{Im} K$, for all $A \in \mathcal{B}, L_{K}^{*} \mathcal{E}_{U} A L_{K}$ (resp. $L_{K}^{*} \mathcal{E}_{V} A L_{K}$ ) is a projector of $\operatorname{Im} K$ and the application $A \in \mathcal{B} \mapsto$ $L_{K}^{*} \mathcal{E}_{U} A L_{K} \in \mathcal{P}(\operatorname{Im} K)$ (resp. $\left.A \in \mathcal{B} \mapsto L_{K}^{*} \mathcal{E}_{V} A L_{K} \in \mathcal{P}(\operatorname{Im} K)\right)$ is the spectral measure associated with $L_{K}^{*} U L_{K}$ (resp. $\left.L_{K}^{*} V L_{K}\right)$. From the previous lemma, $K$ being a commuter of $U$ and $V$, the unitary operators $L_{K}^{*} U L_{K}$ and $L_{K}^{*} V L_{K}$ commute, so, considering a pair $(A, B)$ of elements of $\mathcal{B}, K$ commutes with $\mathcal{E}_{U} A$ and $\mathcal{E}_{V} B$ and the projectors of $\operatorname{Im} K$, $L_{K}^{*} \mathcal{E}_{U} A L_{K}$ and $L_{K}^{*} \mathcal{E}_{V} B L_{K}$, commute. In order to prove that $K$ is a commuter of the projectors $\mathcal{E}_{U} A$ and $\mathcal{E}_{V} B$, it is then sufficient to note that

$$
\begin{aligned}
\left(\mathcal{E}_{U} A\right) K\left(\mathcal{E}_{V} B\right) & =K\left(\mathcal{E}_{U} A\right) K\left(\mathcal{E}_{V} B\right) K=L_{K} L_{K}^{*}\left(\mathcal{E}_{U} A\right) L_{K} L_{K}^{*}\left(\mathcal{E}_{V} B\right) L_{K} L_{K}^{*} \\
& =L_{K} L_{K}^{*}\left(\mathcal{E}_{V} B\right) L_{K} L_{K}^{*}\left(\mathcal{E}_{U} A\right) L_{K} L_{K}^{*}=K\left(\mathcal{E}_{V} B\right) K\left(\mathcal{E}_{U} A\right) K=\left(\mathcal{E}_{V} B\right) K\left(\mathcal{E}_{U} A\right) .
\end{aligned}
$$

Conversely, assume that $K$ is a commuter of the projectors $\mathcal{E}_{U} A$ and $\mathcal{E}_{V} B$, for any pair $(A, B)$ of elements of $\mathcal{B}$. As $K$ commutes with $\mathcal{E}_{U} A$ for all $A \in \mathcal{B}, K$ commutes with $U$. So, $L_{K}^{*} \mathcal{E}_{U} A L_{K}$ is a projector of $\operatorname{Im} K$ and the application $A \in \mathcal{B} \mapsto L_{K}^{*}\left(\mathcal{E}_{U} A\right) L_{K} \in \mathcal{P}(\operatorname{Im} K)$ is the spectral measure associated with the unitary operator $L_{K}^{*} U L_{K}$. The same property stands for $V$, viz. $K V=V K$, and the application $B \in \mathcal{B} \mapsto L_{K}^{*}\left(\mathcal{E}_{V} B\right) L_{K} \in \mathcal{P}(\operatorname{Im} K)$ is the spectral measure 
associated with the unitary operator $L_{K}^{*} V L_{K}$. Let us consider a pair $(A, B)$ of elements of $\mathcal{B}$. As $K$ is a commuter of $\mathcal{E}_{U} A$ and $\mathcal{E}_{V} B$, we can write

$$
L_{K}^{*}\left(\mathcal{E}_{U} A\right) L_{K} L_{K}^{*}\left(\mathcal{E}_{V} B\right) L_{K}=L_{K}^{*}\left(\mathcal{E}_{U} A\right) K\left(\mathcal{E}_{V} B\right) L_{K}=L_{K}^{*}\left(\mathcal{E}_{V} B\right) K\left(\mathcal{E}_{U} A\right) L_{K}=L_{K}^{*}\left(\mathcal{E}_{V} B\right) L_{K} L_{K}^{*}\left(\mathcal{E}_{U} A\right) L_{K} .
$$

Thus the projectors $L_{K}^{*}\left(\mathcal{E}_{U} A\right) L_{K}$ and $L_{K}^{*}\left(\mathcal{E}_{V} B\right) L_{K}$ commute whatever $(A, B) \in \mathcal{B} \times \mathcal{B}$. We conclude that the unitary operators $L_{K}^{*} U L_{K}$ and $L_{K}^{*} V L_{K}$ commute, so (see Lemma 4 ) $K$ is a commuter of the unitary operators $U$ and $V$.

Proposition 2 has the following consequence.

Corollary 1. If $K$ is a commuter of the unitary operators $U$ and $V$, then, for any $(n, m) \in \mathbb{Z}^{2}, K$ is a commuter of the unitary operators $U^{n}$ and $V^{m}$.

Proof. From the background material in Section 2, $\mathcal{E}_{U^{n}}=\widehat{h_{n}} \mathcal{E}_{U}$ and $\mathcal{E}_{V^{m}}=\widehat{h_{m}} \mathcal{E}_{V}$. From Proposition $2, K$ will be a commuter of $U^{n}$ and $V^{m}$ if $K$ is a commuter of the projectors $\widehat{h_{n}} \mathcal{E}_{U} A$ and $\widehat{h_{m}} \mathcal{E}_{V} B$ for all $(A, B) \in \mathcal{B} \times \mathcal{B}$. So, let $(A, B)$ be a pair of elements of $\mathcal{B}$. As $K$ is a commuter of $U$ and $V$, Proposition 2 implies that $K$ is a commuter of the projectors $\mathcal{E}_{U}{\widehat{h_{n}}}^{-1} A$ and $\mathcal{E}_{V}{\widehat{h_{m}}}^{-1} B$, and so of the projectors $\widehat{h_{n}} \mathcal{E}_{U} A$ and $\widehat{h_{m}} \mathcal{E}_{V} B$.

If $U$ and $V$ are two unitary operators, denote by $C$ the projector on the closed vector subspace

$$
\cap_{(n, m) \in \mathbb{Z}^{2}} \operatorname{Ker}\left(U^{n} V^{m}-V^{m} U^{n}\right) .
$$

Proposition 3. $C$ is a commuter of the unitary operators $U$ and $V$.

Proof. First note that $X \in \operatorname{Im} C$ if and only if $U^{n} V^{m} X=V^{m} U^{n} X$ for all $(n, m) \in \mathbb{Z}^{2}$. For any $(p, X) \in \mathbb{Z} \times \operatorname{Im} C$, write

$$
U^{n} V^{m}\left(U^{p} X\right)=U^{n}\left(V^{m} U^{p} X\right)=U^{n}\left(U^{p} V^{m} X\right)=U^{n+p} V^{m} X=V^{m} U^{n+p} X=V^{m} U^{n}\left(U^{p} X\right)
$$

for any $n, m \in \mathbb{Z}$. We can then assert that, for any $X \in H, U C X$ and $U^{-1} C X$ belong to $\operatorname{Im} C$, so $C U C X=U C X$ and $C U^{-1} C X=U^{-1} C X$. Then $C U C=U C$ and $C U^{-1} C=U^{-1} C$. This involves the commutativity of $C$ and $U$, because $C U=\left(U^{-1} C\right)^{*}=\left(C U^{-1} C\right)^{*}=C U C=U C$. Similarly, we can prove that $V C=C V$. Finally, these properties of commutativity and the fact that $C X$ belongs to $\operatorname{Im} C$ allow us to write $U C V X=U V C X=V U C X=V C U X$ whatever $X \in H$. So $U C V=V C U$, which allows us to conclude.

With the same notation, we have the following.

Proposition 4. If $K$ is a commuter of the unitary operators $U$ and $V$, then $K \ll C$.

Proof. Take $X \in \operatorname{Im} K$, i.e., such that $X=K X$. As $K$ is a commuter of the operators $U^{n}$ and $V^{m}$ (see Corollary 1 ), we have

$$
U^{n} V^{m} X=U^{n} V^{m} K X=U^{n} K V^{m} X=V^{m} K U^{n} X=V^{m} U^{n} K X=V^{m} U^{n} X
$$

for any $n, m \in \mathbb{Z}$. Thus $X \in \cap_{(n, m) \in \mathbb{Z}^{2}} \operatorname{Ker}\left(U^{n} V^{m}-V^{m} U^{n}\right)$, i.e., $X \in \operatorname{Im} C$. Hence $\operatorname{Im} K \subset \operatorname{Im} C$, i.e., $K \ll C$.

With these results, we can describe explicitly the maximal commuter of two unitary operators.

Proposition 5. If $U$ and $V$ are two unitary operators of $H$, then $\operatorname{Im} C_{U, V}=\cap_{(n, m) \in \mathbb{Z}^{2}} \operatorname{Ker}\left(U^{n} V^{m}-V^{m} U^{n}\right)$.

The maximal commuter can also be defined by the way of a family of equalizers.

Proposition 6. For any pair $(U, V)$ of unitary operators, we have $C_{U, V}=\inf \left\{R_{V, U^{-n} V U^{n}}: n \in \mathbb{Z}\right\}$.

Proof. By mathematical induction, we can show that for any $m \in \mathbb{N}, U^{-n} V^{m} U^{n}=\left(U^{-n} V U^{n}\right)^{m}$. Then, we extend this property for any $m \in \mathbb{Z}$ : if the property is verified for $m \geq 0$, then when $m<0$, we can write $\left(U^{-n} V U^{n}\right)^{|m|}=$ $U^{-n} V^{|m|} U^{n}$, and taking the adjoint we get $\left(U^{-n} V U^{n}\right)^{-|m|}=U^{-n} V^{-|m|} U^{n}$, i.e., $\left(U^{-n} V U^{n}\right)^{m}=U^{-n} V^{m} U^{n}$. Finally,

$$
\begin{aligned}
\operatorname{Im} C_{U, V} & =\cap_{(n, m) \in \mathbb{Z}^{2}} \operatorname{Ker}\left(U^{n} V^{m}-V^{m} U^{n}\right)=\cap_{n \in \mathbb{Z}} \cap_{m \in \mathbb{Z}} \operatorname{Ker}\left(V^{m}-U^{-n} V^{m} U^{n}\right) \\
& =\cap_{n \in \mathbb{Z}} \cap_{m \in \mathbb{Z}} \operatorname{Ker}\left\{V^{m}-\left(U^{-n} V U^{n}\right)^{m}\right\}=\cap_{n \in \mathbb{Z}} \operatorname{Im} R_{V, U^{-n}} V U^{n}=\operatorname{Im} \inf \left\{R_{V, U^{-n} V U^{n}}: n \in \mathbb{Z}\right\},
\end{aligned}
$$

so the claim holds. 


\subsection{Maximal commuter of a projector and of a unitary operator}

When $A \in \mathcal{L}(H),\left\{A^{n} / n !: n \in \mathbb{N}\right\}$ is a summable family of the $\mathbb{C}$-Banach space $\mathcal{L}(H)$. The sum of this family is denoted $e^{A}$; see [19]. Of course, the formula $e^{A}=\sum_{n \in \mathbb{N}} A^{n} / n$ ! is reminiscent of the formula $e^{z}=\sum_{n \in \mathbb{Z}} z^{n} / n$ !, when $z \in \mathbb{R}$ or $\mathbb{C}$. In the particular case where $P$ is a projector of $H$, we have

$$
e^{i P}=\sum_{n \in \mathbb{N}} \frac{i^{n}}{n !} P^{n}=I+\sum_{n \in \mathbb{N}^{*}} \frac{i^{n}}{n !} P^{n}=I+\left(e^{i}-1\right) P=e^{i} P+P_{\perp} .
$$

It is clear that $e^{i P}$ is a unitary operator with spectral measure $\mathcal{E}_{e^{i P}}=\delta_{0} P_{\perp}+\delta_{1} P$. This yields the following result.

Lemma 5. If $P$ is a projector and $U$ a unitary operator, then $C_{P, U}=C_{e^{i P}, U}$.

Proof. It is easy to check that a projector $K$ is a commuter of $P$ and $U$ if and only if $K$ is commuter of the unitary operators $e^{i P}=I+\left(e^{i}-1\right) P$ and $U$. Therefore, the families of the commuters of $P$ and $U$ and of the commuters of $e^{i P}$ and $U$ are equal, which is why $C_{P, U}=C_{e^{i p}, U}$.

This lemma allows us to use the results of Section 3.2 for the study of the maximal commuter of a projector and a unitary operator.

Proposition 7. For any projector $P$ of $H$ and any unitary operator $U$ of $H$, we have $\operatorname{Im} C_{P, U}=\cap_{n \in \mathbb{Z}} \operatorname{Ker}\left(P U^{n}-U^{n} P\right)$.

Proof. We first notice that for any $m \in \mathbb{Z}$, we have $\left(e^{i P}\right)^{m}=e^{i m} P+P_{\perp}=I+\left(e^{i m}-1\right) P$. This can be proved by induction for any $m \in \mathbb{N}$, and then can be extended to any $m \in \mathbb{Z}$, by using the adjoint form. Then, for any $n, m \in \mathbb{Z}$, we have $\left(e^{i P}\right)^{m} U^{n}-U^{n}\left(e^{i P}\right)^{m}=\left(e^{i m}-1\right)\left(P U^{n}-U^{n} P\right)$. It follows from Proposition 5 that

$$
\operatorname{Im} C_{P, U}=\operatorname{Im} C_{e^{i P}, U}=\cap_{(n, m) \in \mathbb{Z}^{2}} \operatorname{Ker}\left\{\left(e^{i P}\right)^{m} U^{n}-U^{n}\left(e^{i P}\right)^{m}\right\}=\cap_{n \in \mathbb{Z}} \operatorname{Ker}\left(P U^{n}-U^{n} P\right),
$$

which completes the proof.

Noting that $U^{-n} P U^{n}$ is a projector, we have the following ergodic property.

Proposition 8. If $P$ is a projector and $U$ is a unitary operator, then $C_{P, U}=\inf \left\{d\left(P, U^{-n} P U^{n}\right)^{\perp}: n \in \mathbb{Z}\right\}$.

Proof. As $\operatorname{Ker}\left(P U^{n}-U^{n} P\right)=\operatorname{Ker}\left(U^{-n} P U^{n}-P\right)=\operatorname{Im}\left\{d\left(P, U^{-n} P U^{n}\right)^{\perp}\right\}$, we have $\operatorname{Im} C_{P, U}=\cap_{n \in \mathbb{Z}} \operatorname{Im}\left\{d\left(P, U^{-n} P U^{n}\right)^{\perp}\right\}=$ $\operatorname{Im} \inf \left\{d\left(P, U^{-n} P U^{n}\right)^{\perp}: n \in \mathbb{Z}\right\}$, and the property stands.

Remark 1. The maximal commuter $C_{P, U}$ of the projector $P$ and of the unitary operator $U$ measures the level of commutativity between $P$ and $U$. We can easily verify that they commute if and only if $C_{P, U}=I$. As for the formula $C_{P, U}=\inf \left\{d\left(P, U^{-n} P U^{n}\right)^{\perp}: n \in \mathbb{Z}\right\}$, it leads to the following interpretation: if $P U^{n}$ and $U^{n} P$ are close together, that is if the projectors $P$ and $U^{-n} P U^{n}$ are close together, then $d\left(P, U^{-n} P U^{n}\right)$ is of small rank, and so $d\left(P, U^{-n} P U^{n}\right)^{\perp}$ is of high rank, together with the lower bound, $C_{P, U}$, of the family $\left\{d\left(P, U^{-n} P U^{n}\right)^{\perp}: n \in \mathbb{Z}\right\}$.

\subsection{Maximal commuter of two projectors}

If $P$ and $D$ are two projectors, it is easy to check that any commuter of $P$ and $D$ is a commuter of $P$ and $e^{i D}$. Conversely, any commuter of $P$ and $e^{i D}$ is a commuter of $P$ and $D$. Thus, the family of the commuters of $P$ and $D$ coincides with the family of the commuters of $P$ and $e^{i D}$. We deduce the following result.

Lemma 6. If $P$ and $D$ are two projectors, then $C_{P, D}=C_{P, e^{i D}}$.

We can complete this observation with the following.

Proposition 9. If $P$ and $D$ are two projectors, then $\operatorname{Im} C_{P, D}=\operatorname{Ker}(P D-D P)$. 
Proof. For any $n \in \mathbb{Z}$, we have $P\left(e^{i D}\right)^{n}-\left(e^{i D}\right)^{n} P=\left(e^{i n}-1\right)(P D-D P)$. Lemma 6 and Proposition 7 yield Im $C_{P, D}=$ $\operatorname{Im} C_{P, e^{i D}}=\cap_{n \in \mathbb{Z}} \operatorname{Ker}\left\{\left(e^{i n}-1\right)(P D-D P)\right\}=\operatorname{Ker}(P D-D P)$.

Let $(P, D)$ be a pair of projectors. For any $X \in H$, we have

$$
\|P D X-D P X\| \leq\left\|P D X-P D C_{P, D} X\right\|+\left\|P D C_{P, D} X-D P X\right\| .
$$

As $P D C_{P, D} X=P C_{P, D} D X=D C_{P, D} P X=D P C_{P, D} X$, we find

$$
\|P D X-D P X\| \leq\left\|X-C_{P, D} X\right\|+\left\|C_{P, D} X-X\right\|=2\left\|C_{P, D}^{\perp} X\right\|,
$$

which allows us to conclude.

Proposition 10. Given any pair $(P, D)$ of projectors, we have $\|P D X-D P X\| \leq 2\left\|C_{P, D}^{\perp} X\right\|$ for all $X \in H$.

Remark 2. (a) The term "maximal commuter" is justified by the fact that $C_{P, D}$ is the projector on the closed vector subspace of the elements $X$ such that $P D X=D P X$.

(b) The maximal commuter measures the degree of commutativity of two projectors. We can easily check that $C_{P, D}=I$ if and only if $P$ and $D$ commute.

(c) From Proposition 10, when $X$ is close to $\operatorname{Im} C_{P, D}$, i.e., when $\left\|C_{P, D}^{\perp} X\right\|$ is small, then $P D X$ and $D P X$ are close together.

(d) For any pair $(P, D)$ of projectors, $C_{P, D}=C_{P^{\perp}, D}$ because $\operatorname{Ker}(P D-D P)=\operatorname{Ker}\left(P^{\perp} D-D P^{\perp}\right)$.

\subsection{Links between the three families of commuters}

In this section, we will establish and study various relations between the maximal commuters of (a) two unitary operators; (b) a projector and a unitary operator; (c) two projectors. In particular, the maximal commuter of two unitary operators $U$ and $V$ can be expressed through a family of maximal commuters of projectors $\left\{C_{\mathcal{E}_{U} A, \mathcal{E}_{V} B}:(A, B) \in \mathcal{B} \times \mathcal{B}\right\}$.

Proposition 11. If $U$ and $V$ are two unitary operators, we have $C_{U, V}=\inf \left\{C_{\mathcal{E}_{U} A, \mathcal{E}_{V} B}:(A, B) \in \mathcal{B} \times \mathcal{B}\right\}$.

Proof. Let us consider an element $X$ of $\cap_{(A, B) \in \mathcal{B} \times \mathcal{B}} \operatorname{Ker}\left\{\left(\mathcal{E}_{U} A\right)\left(\mathcal{E}_{V} B\right)-\left(\mathcal{E}_{V} B\right)\left(\mathcal{E}_{U} A\right)\right\}$. For any $(B, n) \in \mathcal{B} \times \mathbb{Z}$, we have

$$
Z_{\mathcal{E}_{U}}^{\left(\mathcal{E}_{V} B\right) X}=\left(\mathcal{E}_{V} B\right) \circ Z_{\mathcal{E}_{U}}^{X} .
$$

As $\overline{\operatorname{vect}}\left(1_{A}: A \in \mathcal{B}\right)=L^{2}\left(\Pi, \mathcal{B}, \mu_{Z_{\mathcal{E}_{U}}^{\left(\mathcal{E}_{V} B\right) X}}+\mu_{Z_{\mathcal{E}_{U}}^{X}}\right)$, we can write

$$
e^{i . n}=\lim _{m \rightarrow \infty} \sum_{j=1}^{k_{m}} \alpha_{j, m} 1_{A_{j, m}} \text { in } L^{2}\left(\mu_{Z_{\mathcal{E}_{U}}^{\left(\mathcal{E}_{V} B\right) X}}+\mu_{Z_{\mathcal{E}_{U}}^{X}}\right),
$$

where $A_{j, m} \in \mathcal{B}, \alpha_{j, m} \in \mathbb{C}$ and $k_{m} \in \mathbb{N}^{*}$. Relation (2) is also true in $L^{2}\left(\mu_{Z_{\mathcal{E}_{U}}^{\left(\mathcal{E}_{V} B\right) X}}\right)$ and in $L^{2}\left(\mu_{Z_{\mathcal{E}_{U}}^{X}}\right)$ because

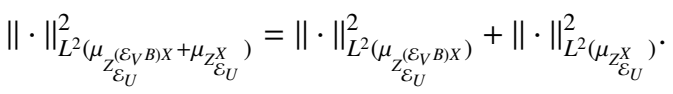

Integrating (2) with respect to the random measure $Z_{\mathcal{E}_{U}}^{X}$ yields

$$
U^{n} X=\lim _{m \rightarrow \infty} \sum_{j=1}^{k_{m}} \alpha_{j, m} Z_{\mathcal{E}_{U}}^{X} A_{j, m} \quad \text { and } \quad\left(\mathcal{E}_{V} B\right) U^{n} X=\lim _{m \rightarrow \infty} \sum_{j=1}^{k_{m}} \alpha_{j, m}\left(\mathcal{E}_{V} B\right) Z_{\mathcal{E}_{U}}^{X} A_{j, m} .
$$

Taking into account (1), we then get

$$
\left(\mathcal{E}_{V} B\right) U^{n} X=\lim _{m \rightarrow \infty} \sum_{j=1}^{k_{m}} \alpha_{j, m} Z_{\mathcal{E}_{U}}^{\left(\mathcal{E}_{V} B\right) X} A_{j, m}
$$


Integrating (2) with respect to the random measure $Z_{\mathcal{E}_{U}}^{\left(\mathcal{E}_{V} B\right) X}$, we find

$$
U^{n}\left(\mathcal{E}_{V} B\right) X=\lim _{m \rightarrow \infty} \sum_{j=1}^{k_{m}} \alpha_{j, m} Z_{\mathcal{E}_{U}}^{\left(\mathcal{E}_{V} B\right) X} A_{j, m} .
$$

Thus, from what precedes, $\left(\mathcal{E}_{V} B\right) U^{n} X=U^{n}\left(\mathcal{E}_{V} B\right) X$ for any $B \in \mathcal{B}$, from which we deduce that $Z_{\mathcal{E}_{V}}^{U^{n}}=U^{n} \circ Z_{\mathcal{E}_{V}}^{X}$. Keeping in mind the background material, we have, for all $n, m \in \mathbb{Z}$,

$$
\int e^{i \cdot m} \mathrm{~d} Z_{\mathcal{E}_{V}}^{U^{n} X}=\int e^{i \cdot m} \mathrm{~d} U^{n} \circ Z_{\mathcal{E}_{V}}^{X}=U^{n}\left(\int e^{i \cdot m} \mathrm{~d} Z_{\mathcal{E}_{V}}^{X}\right)
$$

As $Z_{\mathcal{E}_{V} X}^{U^{n} X}$ and $Z_{\mathcal{E}_{V}}^{X}$ are the random measures respectively associated with the stationary series $\left(V^{m} U^{n} X, m \in \mathbb{Z}\right)$ and $\left(V^{m} X, m \in \mathbb{Z}\right)$, we see that $V^{m} U^{n} X=U^{n} V^{m} X$. This means that $X$ belongs to $\operatorname{Ker}\left(V^{m} U^{n}-U^{n} V^{m}\right)$ whatever $n, m \in \mathbb{Z}$, so that $X \in \cap_{(n, m) \in \mathbb{Z}^{2}} \operatorname{Ker}\left(V^{m} U^{n}-U^{n} V^{m}\right)=\operatorname{Im} C_{U, V}$. We have just proved that

$$
\cap_{(A, B) \in \mathcal{B} \times \mathcal{B}} \operatorname{Ker}\left\{\left(\mathcal{E}_{U} A\right)\left(\mathcal{E}_{V} B\right)-\left(\mathcal{E}_{V} B\right)\left(\mathcal{E}_{U} A\right)\right\} \subset \operatorname{Im} C_{U, V}
$$

and hence

$$
\operatorname{Im} \inf \left\{C_{\mathcal{E}_{U} A, \mathcal{E}_{V} B}:(A, B) \in \mathcal{B} \times \mathcal{B}\right\} \subset \operatorname{Im} C_{U, V},
$$

so that $\inf \left\{C_{\mathcal{E}_{U} A, \mathcal{E}_{V} B}:(A, B) \in \mathcal{B} \times \mathcal{B}\right\} \ll C_{U, V}$.

As $C_{U, V}$ is a commuter of $U$ and $V$, Proposition 2 implies that it is a commuter of the projectors $\mathcal{E}_{U} A$ and $\mathcal{E}_{V} B$ for all $(A, B) \in \mathcal{B} \times \mathcal{B}$. So we have $C_{U, V} \ll C_{\mathcal{E}_{U} A, \mathcal{E}_{V} B}$. This means that $C_{U, V}$ is a lower bound of the family of projectors $\left\{C_{\mathcal{E}_{U} A, \mathcal{E}_{V} B}:(A, B) \in \mathcal{B} \times \mathcal{B}\right\}$. Therefore, $C_{U, V} \ll \inf \left\{C_{\mathcal{E}_{U} A, \mathcal{E}_{V} B}:(A, B) \in \mathcal{B} \times \mathcal{B}\right\}$, which allows us to conclude.

Let us examine what this last result becomes in the particular case where $U=e^{i P}, P$ being a projector.

Proposition 12. If $P$ is a projector and $V$ a unitary operator of $H$, then we have $C_{P, V}=\inf \left\{C_{P, \mathcal{E}_{V} B}: B \in \mathcal{B}\right\}$.

Proof. We know that $\mathcal{E}_{e^{i P}}=\delta_{0} P_{\perp}+\delta_{1} P$. Depending on whether an element $A$ of $\mathcal{B}$ includes 0 and 1,0 and not 1,1 and not 0 , or neither 0 neither $1, \mathcal{E}_{e^{i P}} A$ takes the value $I, P_{\perp}, P$ or 0 , respectively. Thus, for any $A, B \in \mathcal{B}, C_{\mathcal{E}_{e^{i P}} A, \mathcal{E}_{V} B}$

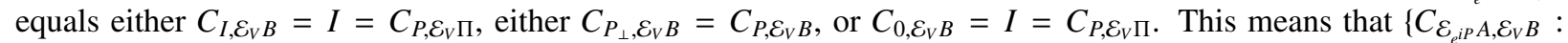
$(A, B) \in \mathcal{B} \times \mathcal{B}\} \subset\left\{C_{P, \mathcal{E}_{V} B}: B \in \mathcal{B}\right\}$. However, we also have $\left\{C_{P, \mathcal{E}_{V} B}: B \in \mathcal{B}\right\} \subset\left\{C_{\mathcal{E}_{e^{i P}} A, \mathcal{E}_{V} B}:(A, B) \in \mathcal{B} \times \mathcal{B}\right\}$, because $C_{P, \mathcal{E}_{V} B}=C_{\mathcal{E}_{e i}(\{1\}), \mathcal{E}_{V} B}$. Thus we can write $\left\{C_{\mathcal{E}_{e} i P, \mathcal{E}_{V} B}:(A, B) \in \mathcal{B} \times \mathcal{B}\right\}=\left\{C_{P, \mathcal{E}_{V} B}: B \in \mathcal{B}\right\}$. In view of Lemma 6 and

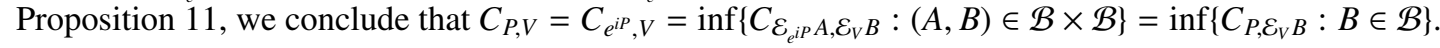

The following is a consequence of Proposition 12.

Corollary 2. If $U$ and $V$ are any two unitary operators, then $C_{U, V}=\inf \left\{C_{\mathcal{E}_{V} B, U}: B \in \mathcal{B}\right\}$.

Proof. From Propositions 11 and 12, we get

$$
\begin{aligned}
\operatorname{Im} C_{U, V} & =\cap_{B \in \mathcal{B}} \cap_{A \in \mathcal{B}} \operatorname{Im} C_{\mathcal{E}_{U} A, \mathcal{E}_{V} B}=\cap_{B \in \mathcal{B}} \operatorname{Im} \inf \left\{C_{\mathcal{E}_{U} A, \mathcal{E}_{V} B}: A \in \mathcal{B}\right\} \\
& =\cap_{B \in \mathcal{B}} \operatorname{Im} C_{\mathcal{E}_{V} B, U}=\operatorname{Im} \inf \left\{C_{\mathcal{E}_{V} B, U}: B \in \mathcal{B}\right\},
\end{aligned}
$$

which completes the proof.

In general, if $U$ and $V$ are two unitary operators which do not commute, the series $\left(U^{n} V^{m} X,(n, m) \in \mathbb{Z}^{2}\right)$ is not stationary. Nevertheless, if $X$ belongs to $\operatorname{Im} C_{U, V}$, i.e., if $U^{n} V^{m} X=V^{m} U^{n} X$ for any $n, m \in \mathbb{Z}$, then for any pair $\left((n, m),\left(n^{\prime}, m^{\prime}\right)\right)$ of elements of $\mathbb{Z}^{2}$, we have $<U^{n} V^{m} X, U^{n^{\prime}} V^{m^{\prime}} X>=<U^{n-n^{\prime}} V^{m} X, V^{m^{\prime}} X>=<V^{m} U^{n-n^{\prime}} X, V^{m^{\prime}} X>=$ $<V^{m-m^{\prime}} U^{n-n^{\prime}} X, X>=<U^{n-n^{\prime}} V^{m-m^{\prime}} X, X>$. So we get the following result.

Proposition 13. Let $U$ and $V$ be two unitary operators. Then, for any $X \in \operatorname{Im} C_{U, V}$, the series $\left(U^{n} V^{m} X,(n, m) \in \mathbb{Z}^{2}\right)$ and $\left(V^{m} U^{n} X,(n, m) \in \mathbb{Z}^{2}\right)$ are equal and stationary. 
What can be said if $X$ is close to $\operatorname{Im} C_{U, V}$ ? The following proposition gives a partial answer to this question.

Proposition 14. Let $U$ and $V$ be two unitary operators. Then, for any $X \in H$, we have

(i) $\left\|\left(\mathcal{E}_{U} A\right)\left(\mathcal{E}_{V} B\right) X-\left(\mathcal{E}_{V} B\right)\left(\mathcal{E}_{U} A\right) X\right\| \leq 2\left\|C_{U, V}^{\perp} X\right\|$, for all $A, B \in \mathcal{B}$.

(ii) $\left\|U^{n} V^{m} X-V^{m} U^{n} X\right\| \leq 2\left\|C_{U, V}^{\perp} X\right\|$ for all $n, m \in \mathbb{Z}$.

(iii) $\left|<U^{n} V^{m} X, U^{n^{\prime}} V^{m^{\prime}} X>-<U^{n-n^{\prime}} V^{m-m^{\prime}} X, X>\right| \leq 4\left\|C_{U, V}^{\perp} X\right\|\|X\|$, for all $n, m, n^{\prime}, m^{\prime} \in \mathbb{Z}$.

Proof. From Proposition 11, we have $C_{\mathcal{E}_{U} A, \mathcal{E}_{V} B}^{\perp} \ll C_{U, V}^{\perp}$. Proposition 10 then allows us to get Part (i), viz.

$$
\left\|\left(\mathcal{E}_{U} A\right)\left(\mathcal{E}_{V} B\right) X-\left(\mathcal{E}_{V} B\right)\left(\mathcal{E}_{U} A\right) X\right\| \leq 2\left\|C_{\mathcal{E}_{U} A, \mathcal{E}_{V} B} X\right\| \leq 2\left\|C_{U, V}^{\perp} X\right\|
$$

Turning to Part (ii), for any $X \in H, C_{U, V} X$ belongs to $\cap_{(n, m) \in \mathbb{Z}^{2}} \operatorname{Ker}\left(U^{n} V^{m}-V^{m} U^{n}\right)$ and then $U^{n} V^{m} C_{U, V} X=V^{m} U^{n} C_{U, V} X$. We can then write

$$
\begin{aligned}
\left\|U^{n} V^{m} X-V^{m} U^{n} X\right\| & \leq\left\|U^{n} V^{m} X-U^{n} V^{m} C_{U, V} X\right\|+\left\|U^{n} V^{m} C_{U, V} X-V^{m} U^{n} X\right\| \\
& =\left\|X-C_{U, V} X\right\|+\left\|V^{m} U^{n} C_{U, V} X-V^{m} U^{n} X\right\|=\left\|C_{U, V}^{\perp} X\right\|+\left\|C_{U, V} X-X\right\|=2\left\|C_{U, V}^{\perp} X\right\| .
\end{aligned}
$$

As for Part (iii), it is a consequence of Part (ii). Indeed, for any pair $\left((n, m),\left(n^{\prime}, m^{\prime}\right)\right)$ of elements of $\mathbb{Z}^{2}$, we have

$$
\begin{aligned}
\mid<U^{n} V^{m} X, U^{n^{\prime}} V^{m^{\prime}} X & >-<U^{n-n^{\prime}} V^{m-m^{\prime}} X, X>\mid \\
=\mid & <V^{m-m^{\prime}} U^{n-n^{\prime}} X-U^{n-n^{\prime}} V^{m-m^{\prime}} X, X>+<U^{n-n^{\prime}} V^{m} X-V^{m} U^{n-n^{\prime}} X, V^{m^{\prime}} X>\mid \\
& \leq\left\|V^{m-m^{\prime}} U^{n-n^{\prime}} X-U^{n-n^{\prime}} V^{m-m^{\prime}} X\left|\|\mid\|+\left\|U^{n-n^{\prime}} V^{m} X-V^{m} U^{n-n^{\prime}} X\right\|\left\|V^{m^{\prime}} X\right\| \leq 4\left\|C_{U, V}^{\perp} X\right\|\|X\| .\right.\right.
\end{aligned}
$$

Thus the proof is complete.

Remark 3. (a) Parts (i) and (ii) of Proposition 14 respectively concern the frequential and temporal expressions of the same phenomenon: the proximity between $X$ and $\operatorname{Im} C_{U, V}$.

(b) If $X$ is close to $\operatorname{Im} C_{U, V}$, i.e., if $\left\|C_{U, V}^{\perp} X\right\|$ is small, then the series $\left(U^{n} V^{m} X,(n, m) \in \mathbb{Z}^{2}\right)$ is almost stationary: for any $n, m, n^{\prime}, m^{\prime} \in \mathbb{Z}^{2},\left|<U^{n} V^{m} X, U^{n^{\prime}} V^{m^{\prime}} X>-<U^{n-n^{\prime}} V^{m-m^{\prime}} X, X>\right|$ is small.

(c) For any $\left(X, X^{\prime}\right) \in H \times \operatorname{Im} C$, we have the following commutativity property:

$$
\left\|U^{n} V^{m} X^{\prime}-U^{n} V^{m} X\right\|=\left\|X^{\prime}-X\right\|=\left\|V^{m} U^{n} X^{\prime}-V^{m} U^{n} X\right\|
$$

for any $n, m \in \mathbb{Z}$. Recall that the series $\left(U^{n} V^{m} X^{\prime},(n, m) \in \mathbb{Z}^{2}\right)=\left(V^{m} U^{n} X^{\prime},(n, m) \in \mathbb{Z}^{2}\right)$ is stationary.

(d) It can be checked that $C_{U, V}$ is an equalizer of $U V$ and $V U$. If $R$ denotes the maximal equalizer of the unitary operators $U V$ and $V U$, then $d\left(\mathcal{E}_{U V} A, \mathcal{E}_{V U} A\right) \ll E_{\mathcal{E}_{U V}, \mathcal{E}_{V U}}=R^{\perp} \ll C_{U, V}^{\perp}$. Note that this equalizer can be different from the maximal equalizer.

Of course, we can obtain similar results to the last proposition for the maximal commuter of a projector and a unitary operator.

Corollary 3. If $P$ is a projector and $V$ a unitary operator, then, for any $X \in H$, we have

(i) $\left\|P\left(\mathcal{E}_{V} B\right) X-\left(\mathcal{E}_{V} B\right) P X\right\| \leq 2\left\|C_{P, V}^{\perp} X\right\|$ for all $B \in \mathcal{B}$.

(ii) $\left\|P V^{m} X-V^{m} P X\right\| \leq\left\|C_{P, V}^{\perp} X\right\| /|\sin (1)|$ for all $m \in \mathbb{Z}$. 
Proof. We use Proposition 14 in the particular case where $U=e^{i P}=e^{i} P+P^{\perp}=I+\left(e^{i}-1\right) P$. We recall that $\mathcal{E}_{e^{i P}}=\delta_{0} P^{\perp}+\delta_{1} P$. Part (i) of this proposition implies that, for any $B \in \mathcal{B}$,

$$
\left\|P\left(\mathcal{E}_{V} B\right) X-\left(\mathcal{E}_{V} B\right) P X\right\|=\left\|\mathcal{E}_{e^{i P}}(\{1\})\left(\mathcal{E}_{V} B\right) X-\left(\mathcal{E}_{V} B\right) \mathcal{E}_{e^{i P}}(\{1\}) X\right\| \leq 2\left\|C_{e^{i p}, V}^{\perp} X\right\|=2\left\|C_{P, V}^{\perp} X\right\|,
$$

which proves Part (i). As for Part (ii), it can be deduced from Part (ii) of Proposition 14, which implies

$$
\left\|\left(e^{i 2}-1\right)\left(P V^{m}-V^{m} P\right) X\right\|=\left\|U^{2} V^{m} X-V^{m} U^{2} X\right\| \leq 2\left\|C_{e^{i p}, V}^{\perp} X\right\|=2\left\|C_{P, V}^{\perp} X\right\| .
$$

Thus, for any $m \in \mathbb{Z},\left\|P V^{m} X-V^{m} P X\right\| \leq\left\|C_{P, V}^{\perp} X\right\| /|\sin (1)|$ and hence the argument is complete.

Remark 4. These inequalities are respectively the temporal and the frequential expressions of the same phenomenon. They can be interpretated as follows: when $X$ is close to $\operatorname{Im} C_{P, V}$, that is when $\left\|C_{P, V}^{\perp} X\right\|$ is small, then $\left(P V^{m} X, m \in \mathbb{Z}\right)$ is an almost stationary series, close to the series $\left(V^{m} P X, m \in \mathbb{Z}\right)$. As for $P \circ Z_{\mathcal{E}_{y}}^{X}$, it is almost a random measure, close to $Z_{\mathcal{E}_{V}}^{P X}$, which is the random measure associated with the stationary series $\left(V^{m} P X, m \in \mathbb{Z}\right)$.

\subsection{Commuter and history of a series}

When $\left(X_{n}, n \in \mathbb{Z}\right.$ ) is a stationary series (of elements of $H$ ), we call $P_{n}$ the projector on $\overline{\operatorname{vect}}\left\{X_{m}: m \leq n\right\}$, as per $[11,17]$. This projector $P_{n}$ can be called the projector on the history to time $n$. Obviously, if $n^{\prime} \leq n$, from $\overline{\operatorname{vect}}\left\{X_{m}: m \leq n^{\prime}\right\} \subset \overline{\operatorname{vect}}\left\{X_{m}: m \leq n\right\}$, we have $P_{n^{\prime}} \ll P_{n}$. It can be shown [11] that the applications $P_{-\infty}: X \in H \mapsto$ $\lim _{n \rightarrow \infty} P_{-n} X \in H$ and $P_{+\infty}: X \in H \mapsto \lim _{n \rightarrow \infty} P_{n} X \in H$ are projectors.

From the property $P_{n^{\prime}} \ll P_{n}$ when $n^{\prime} \leq n$, we deduce that the sequences of projectors $\left(P_{k+n}, n \in \mathbb{N}\right)$ and $\left(P_{k-n}, n \in \mathbb{N}\right)$ are increasing and decreasing, respectively. In view of the background material in Section 2, we can write $\lim _{n}^{r} P_{k+n}=\sup \left\{P_{k+n}: n \in \mathbb{N}\right\}$ and $\lim _{n}^{r} P_{k-n}=\inf \left\{P_{k-n}: n \in \mathbb{N}\right\}$. Noting that $\sup \left\{P_{k+n}: n \in \mathbb{N}\right\}=\sup \left\{P_{n}: n \in \mathbb{Z}\right\}$ and $\inf \left\{P_{k-n}: n \in \mathbb{N}\right\}=\inf \left\{P_{n}: n \in \mathbb{Z}\right\}$, one can then make the following assertion:

For any $k \in \mathbb{Z}$, the sequence $\left(P_{k+n}, n \in \mathbb{N}\right) r$-converges increasingly to $\sup \left\{P_{n}: n \in \mathbb{Z}\right\}$, and the sequence $\left(P_{k-n}, n \in \mathbb{N}\right) r$-converges decreasingly to $\inf \left\{P_{n}: n \in \mathbb{Z}\right\}$.

Thus, when $k=0, \lim _{n}^{r} P_{n}=\sup \left\{P_{n}: n \in \mathbb{Z}\right\}$ and $\lim _{n}^{r} P_{-n}=\inf \left\{P_{n}: n \in \mathbb{Z}\right\}$. As $r$-convergence implies point-wise convergence, on one hand we have $\lim _{n} P_{n} X=\left(\sup \left\{P_{n}: n \in \mathbb{Z}\right\}\right) X$ and hence $P_{+\infty}=\sup \left\{P_{n}: n \in \mathbb{Z}\right\}$. Furthermore, $\lim _{n} P_{-n} X=\left(\inf \left\{P_{n}: n \in \mathbb{Z}\right\}\right) X$ and hence $P_{-\infty}=\inf \left\{P_{n}: n \in \mathbb{Z}\right\}$.

Let $U$ be a unitary operator of $H$ such that $U X_{n}=X_{n+1}$ for all $n \in \mathbb{Z}$. There exists at least one $U$. If $H=\overline{\operatorname{vect}}\left\{X_{n}\right.$ : $n \in \mathbb{Z}\}$, then $U$ is unique, and is often named the shift operator. So we have the following property.

Lemma 7. For any $k \in \mathbb{Z}, C_{P_{k}, U}=\inf \left\{d\left(P_{k}, P_{k+n}\right)^{\perp}: n \in \mathbb{Z}\right\}$.

Proof. As stated in [11], $U^{n}\left(\operatorname{Im} P_{k}\right)=\operatorname{Im} P_{k+n}$. Hence, $U^{n} P_{k}=P_{k+n} U^{n} P_{k}$ for any $(n, k) \in \mathbb{Z}^{2}$. We also can write $U^{-n} P_{k+n}=P_{k} U^{-n} P_{k+n}$, because $(-n, k+n) \in \mathbb{Z}^{2}$. This implies, taking the adjoint, that $P_{k+n} U^{n}=P_{k+n} U^{n} P_{k}$. So we have $P_{k+n} U^{n}=U^{n} P_{k}$ or otherwise $P_{k+n}=U^{n} P_{k} U^{-n}$. Proposition 8 then implies

$$
C_{P_{k}, U}=\inf \left\{d\left(P_{k}, U^{-n} P_{k} U^{n}\right)^{\perp}: n \in \mathbb{Z}\right\}=\inf \left\{d\left(P_{k}, U^{n} P_{k} U^{-n}\right)^{\perp}: n \in \mathbb{Z}\right\}=\inf \left\{d\left(P_{k}, P_{k+n}\right)^{\perp}: n \in \mathbb{Z}\right\},
$$

whence the proof is complete.

We are now in a position to state the main result of this section.

Proposition 15. For any $k \in \mathbb{Z}$, we have $C_{P_{k}, U}=P_{+\infty}^{\perp}+P_{-\infty}$.

Proof. Let $X$ be an element of $\operatorname{Im} C_{P_{k}, U}$, i.e., of $\cap_{n \in \mathbb{Z}} \operatorname{Im} d\left(P_{k}, P_{k+n}\right)^{\perp}=\cap_{n \in \mathbb{Z}} \operatorname{Ker}\left(P_{k}-P_{k+n}\right)$. This means that $P_{k} X=$ $P_{k+n} X$ for all $n \in \mathbb{Z}$. So, as $\lim _{n}^{r} P_{k+n}=P_{+\infty}$,

$$
P_{+\infty} X=\lim _{n \rightarrow \infty} P_{k+n} X=P_{k} X
$$

In a similar way, we have $P_{k} X=P_{k+n} X$ for all $n \in \mathbb{Z}$, and hence $P_{k} X=P_{k-n} X$ for all $n \in \mathbb{Z}$. Therefore, as $\lim _{n}^{r} P_{k-n}=P_{-\infty}$,

$$
P_{-\infty} X=\lim _{n \rightarrow \infty} P_{k-n} X=P_{k} X .
$$


Combining (3) and (4), we have $P_{-\infty} X=P_{+\infty} X$, which brings us to conclude that $X$ belongs to $\operatorname{Ker}\left(P_{+\infty}-P_{-\infty}\right)=$ $\operatorname{Im}\left(P_{+\infty}-P_{-\infty}\right)^{\perp}=\operatorname{Im}\left(P_{+\infty}^{\perp}+P_{-\infty}\right)$. Thus we have proved that $\operatorname{Im}\left(C_{P_{k}, U}\right) \subset \operatorname{Im}\left(P_{+\infty}^{\perp}+P_{-\infty}\right)$.

Conversely, consider an element $X$ of $\operatorname{Im}\left(P_{+\infty}^{\perp}+P_{-\infty}\right)=\operatorname{Ker}\left(P_{+\infty}-P_{-\infty}\right)$. Then $P_{+\infty} X=P_{-\infty} X$. Fix $m \in \mathbb{Z}$. From $P_{-\infty}=\inf \left\{P_{n}: n \in \mathbb{Z}\right\} \ll P_{m} \ll \sup \left\{P_{n}: n \in \mathbb{Z}\right\}=P_{+\infty}$, we deduce that $\left\|P_{-\infty} X\right\| \leq\left\|P_{m} X\right\| \leq\left\|P_{+\infty} X\right\|$. Given that $\left\|P_{-\infty} X\right\|=\left\|P_{+\infty} X\right\|,\left\|P_{m} X\right\|=\left\|P_{-\infty} X\right\|$, we have $P_{-\infty} X=P_{m} X$. Indeed,

$$
\left\|P_{m} X\right\|^{2}=\left\|\left\{P_{-\infty}+\left(P_{m}-P_{-\infty}\right)\right\} X\right\|^{2}=\left\|P_{-\infty} X\right\|^{2}+\left\|\left(P_{m}-P_{-\infty}\right) X\right\|^{2} .
$$

As this is true for arbitrary $m \in \mathbb{Z}$, we have $P_{-\infty} X=P_{k} X=P_{k+n} X$ for all $n \in \mathbb{Z}$. We can thus conclude that $X$ belongs to $\cap_{n \in \mathbb{Z}} \operatorname{Ker}\left(P_{k}-P_{k+n}\right)=\cap_{n \in \mathbb{Z}} \operatorname{Im} d\left(P_{k}, P_{k+n}\right)^{\perp}=\operatorname{Im} C_{P_{k}, U}$. Hence, $\operatorname{Im}\left(P_{+\infty}^{\perp}+P_{-\infty}\right) \subset \operatorname{Im} C_{P_{k}, U}$. This, combined with the previous inclusion, gives $\operatorname{Im} C_{P_{k}, U}=\operatorname{Im}\left(P_{+\infty}^{\perp}+P_{-\infty}\right)$, and finally we have $C_{P_{k}, U}=P_{+\infty}^{\perp}+P_{-\infty}$.

Remark 5. From $[11,17]$, the series $\left(X_{n}, n \in \mathbb{Z}\right)$ is said to be regular or purely non-deterministic when $P_{-\infty}=0$, and to be singular or purely deterministic when $P_{-\infty}=P_{+\infty}$. In order to simplify these definitions, let us consider the case where $H=\overline{\operatorname{vect}}\left(X_{n}, n \in \mathbb{Z}\right)$. So $P_{+\infty}=I$. The series then will be said to be singular when $P_{-\infty}=I$. As for the formula $C_{P_{k}, U}=P_{+\infty}^{\perp}+P_{-\infty}$, it becomes $C_{P_{k}, U}=P_{-\infty}: P_{-\infty}$ is the maximal commuter of the projector $P_{k}$ and the unitary operator $U$. This gives us a new definition of the projector $P_{-\infty}$, and makes the maximal commuter relevant once again. Then we can assert that the series is singular if and only if $C_{P_{k}, U}=I$, i.e., if and only if the projector on the history to the instant $k$ and the shift operator commute.

\section{Relations between the spectral measures $\mathcal{E}_{U}, \mathcal{E}_{V}, \mathcal{E}_{U V}$ and $\mathcal{E}_{V U}$}

When two unitary operators $U$ and $V$ commute, we can compute the spectral measure associated with $U V=V U$, thanks to the convolution product of the spectral measures respectively associated with $U$ and $V$. Now what happens when the operators do not commute? The maximal commuter will help us to address this question. Let us first look at some preliminary results.

Lemma 8. If $P$ and $Q$ are two projectors on $H$ which commute, then $L_{P}^{*} Q L_{P}$ is a projector of $\operatorname{Im} P$.

Proof. This result holds because $\left(L_{P}^{*} Q L_{P}\right)^{*}=L_{P}^{*} Q L_{P}$ and $\left(L_{P}^{*} Q L_{P}\right)\left(L_{P}^{*} Q L_{P}\right)=L_{P}^{*} Q P Q L_{P}=L_{P}^{*} P Q Q L_{P}=L_{P}^{*} Q L_{P}$.

Lemma 9. If $P$ is a projector of $H$ and $Q$ a projector of $\operatorname{Im} P$, then $L_{P} Q L_{P}^{*}$ is a projector of $H$.

Proof. This is because $L_{P} Q L_{P}^{*}$ is self-adjoint and $L_{P} Q L_{P}^{*} L_{P} Q L_{P}^{*}=L_{P} Q I_{\operatorname{Im} P} Q L_{P}^{*}=L_{P} Q Q L_{P}^{*}=L_{P} Q L_{P}^{*}$.

Lemma 10. If $C$ is a projector of $H$ which commutes with the projectors $P$ and $P^{\prime}$, then $\inf \left(L_{C}^{*} P L_{C}, L_{C} P^{\prime} L_{C}^{*}\right)=$ $L_{C}^{*} \inf \left(P, P^{\prime}\right) L_{C}$.

Proof. As $C$ commutes with each of the elements of the family of projectors $\left\{P, P^{\prime}\right\}$, it commutes with $\inf \left(P, P^{\prime}\right)$. As $C$ commutes with the projectors $P, P^{\prime}$ and $\inf \left(P, P^{\prime}\right)$, from Lemma $8, L_{C}^{*} P L_{C}, L_{C}^{*} P^{\prime} L_{C}$, and $L_{C}^{*} \inf \left(P, P^{\prime}\right) L_{C}$ are projectors of $\operatorname{Im} C$. Given that

$$
L_{C}^{*} \inf \left(P, P^{\prime}\right) L_{C} L_{C}^{*} P L_{C}=L_{C}^{*} \inf \left(P, P^{\prime}\right) C P L_{C}=L_{C}^{*} C \inf \left(P, P^{\prime}\right) P L_{C}=L_{C}^{*} \inf \left(P, P^{\prime}\right) L_{C},
$$

we deduce that $L_{C}^{*} \inf \left(P, P^{\prime}\right) L_{C} \ll L_{C}^{*} P L_{C}$. In the same way, we establish that $L_{C}^{*} \inf \left(P, P^{\prime}\right) L_{C} \ll L_{C}^{*} P^{\prime} L_{C}$, so that $L_{C}^{*} \inf \left(P, P^{\prime}\right) L_{C}$ is a lower bound of the family of projectors $\left\{L_{C}^{*} P L_{C}, L_{C}^{*} P^{\prime} L_{C}\right\}$.

Let us consider a lower bound $K$ of the family $\left\{L_{C}^{*} P L_{C}, L_{C}^{*} P^{\prime} L_{C}\right\}$. From Lemma $9, L_{C} K L_{C}^{*}$ is a projector of $H$. From $K \ll L_{C}^{*} P L_{C}$, we deduce that $K L_{C}^{*} P L_{C}=K$ and hence $L_{C} K L_{C}^{*}=L_{C} K L_{C}^{*} P L_{C} L_{C}^{*}=L_{C} K L_{C}^{*} P C=L_{C} K L_{C}^{*} C P=$ $L_{C} K L_{C}^{*} P$. This means that $L_{C}^{*} K L_{C} \ll P$. In the same way, we can show that $L_{C} K L_{C}^{*} \ll P^{\prime}$ and hence $L_{C} K L_{C}^{*}$ is a lower bound of the family $\left\{P, P^{\prime}\right\}$, so $L_{C} K L_{C}^{*} \ll \inf \left(P, P^{\prime}\right)$. Then we can write $L_{C}^{*} L_{C} K L_{C}^{*} \inf \left(P, P^{\prime}\right) L_{C}=L_{C}^{*} L_{C} K L_{C}^{*} L_{C}$, that is $K L_{C}^{*} \inf \left(P, P^{\prime}\right) L_{C}=K$. This means that $L_{C}^{*} \inf \left(P, P^{\prime}\right) L_{C}$ is the largest lower bound of the family of projectors $\left\{L_{C}^{*} P L_{C}, L_{C}^{*} P^{\prime} L_{C}\right\}$. Thus the proof is complete.

In what follows, $U$ and $V$ are two unitary operators, and we denote by $C$ their maximal commuter. As $C$ commutes with $U$ and $V$, it follows from the background material in Section 2 that $U^{\prime}=L_{C}^{*} U L_{C}$ and $V^{\prime}=L_{C}^{*} V L_{C}$ are unitary operators of $\operatorname{Im} C$. Moreover, $\mathcal{E}_{U^{\prime}} A=L_{C}^{*}\left(\mathcal{E}_{U} A\right) L_{C}$ and $\mathcal{E}_{V^{\prime}} A=L_{C}^{*}\left(\mathcal{E}_{V} A\right) L_{C}$ for all $A \in \mathcal{B}$. As $C$ is a commuter of the unitary operators $U$ and $V$, the unitary operators $U^{\prime}$ and $V^{\prime}$ commute (see Lemma 4), yielding the following result. 
Proposition 16. There exists a spectral measure $\mathcal{E}_{U^{\prime}} \otimes \mathcal{E}_{V^{\prime}}$, and only one, on $\mathcal{B} \otimes \mathcal{B}$ for $\operatorname{Im} C$, such that $\mathcal{E}_{U^{\prime}} \otimes \mathcal{E}_{V^{\prime}}(A \times B)=$ $L_{C}^{*} \inf \left(\mathcal{E}_{U} A, \mathcal{E}_{V} B\right) L_{C}$ for all $A, B \in \mathcal{B}$.

Proof. As $U^{\prime} V^{\prime}=V^{\prime} U^{\prime}$, the spectral measures $\mathcal{E}_{U^{\prime}}$ and $\mathcal{E}_{V^{\prime}}$ commute and we can consider $\mathcal{E}_{U^{\prime}} \otimes \mathcal{E}_{V^{\prime}}$, which is a spectral measure on $\mathcal{B} \otimes \mathcal{B}$ for $\operatorname{Im} C$ such that, for all $A, B \in \mathcal{B}$,

$$
\mathcal{E}_{U^{\prime}} \otimes \mathcal{E}_{V^{\prime}}(A \times B)=\left(\mathcal{E}_{U^{\prime}} A\right)\left(\mathcal{E}_{V^{\prime}} B\right)=L_{C}^{*}\left(\mathcal{E}_{U} A\right) L_{C} L_{C}^{*}\left(\mathcal{E}_{V} B\right) L_{C}=\inf \left\{L_{C}^{*}\left(\mathcal{E}_{U} A\right) L_{C}, L_{C}^{*}\left(\mathcal{E}_{V} B\right) L_{C}\right\}
$$

As $C$ commutes with $U$ and $V$, it commutes with the projectors $\mathcal{E}_{U} A$ and $\mathcal{E}_{V} B$. So we can use Lemma 10 , and write

$$
\inf \left\{L_{C}^{*}\left(\mathcal{E}_{U} A\right) L_{C}, L_{C}^{*}\left(\mathcal{E}_{V} B\right) L_{C}\right\}=L_{C}^{*} \inf \left(\mathcal{E}_{U} A, \mathcal{E}_{V} B\right) L_{C},
$$

which leads us to conclude that the spectral measure $\mathcal{E}_{U^{\prime}} \otimes \mathcal{E}_{V^{\prime}}$ is the only one on $\mathcal{B} \otimes \mathcal{B}$ for $\operatorname{Im} C$ such that, for all $A, B \in \mathcal{B}, \mathcal{E}_{U^{\prime}} \otimes \mathcal{E}_{V^{\prime}}(A \times B)=L_{C}^{*} \inf \left(\mathcal{E}_{U} A, \mathcal{E}_{V} B\right) L_{C}$.

Remark 6. When $U$ and $V$ commute, we know how to define a spectral measure on $\mathcal{B} \otimes \mathcal{B}$ for $H$. When $U$ and $V$ do not commute, we know how to define a spectral measure on $\mathcal{B} \otimes \mathcal{B}$, but for $\operatorname{Im} C$, which is a closed vector subspace of $H$. Of course, when $U$ and $V$ commute, we recover the definition of the spectral measure $\mathcal{E}_{U} \otimes \mathcal{E}_{V}$ such that $\mathcal{E}_{U} \otimes \mathcal{E}_{V}(A \times B)=\inf \left(\mathcal{E}_{U} A, \mathcal{E}_{V} B\right)=\left(\mathcal{E}_{U} A\right)\left(\mathcal{E}_{V} B\right)$ for all $A, B \in \mathcal{B}$.

Now we are going to study some relations between the spectral measures $\mathcal{E}_{U V}, \mathcal{E}_{V U}$ and $\mathcal{E}_{U^{\prime}} * \mathcal{E}_{V^{\prime}}=\mathcal{E}_{U^{\prime} V^{\prime}}=$ $\mathcal{E}_{V^{\prime} U^{\prime}}=S\left(\mathcal{E}_{U^{\prime}} \otimes \mathcal{E}_{V^{\prime}}\right)$.

Proposition 17. For any $A \in \mathcal{B}$, we have

(i) $L_{C} \circ\left\{\mathcal{E}_{U^{\prime}} * \mathcal{E}_{V^{\prime}}(A)\right\} \circ L_{C}^{*}=C \mathcal{E}_{U V} A=C \mathcal{E}_{V U} A=C \inf \left(\mathcal{E}_{U V} A, \mathcal{E}_{V U} A\right)=\inf \left(C, \mathcal{E}_{U V} A, \mathcal{E}_{V U} A\right)$.

(ii) $L_{C} \circ\left\{\mathcal{E}_{U^{\prime}} * \mathcal{E}_{V^{\prime}}(A)\right\} \circ L_{C}^{*} \ll \inf \left(\mathcal{E}_{U V} A, \mathcal{E}_{V U} A\right) \ll \sup \left(\mathcal{E}_{U V} A, \mathcal{E}_{V U} A\right) \ll C^{\perp}+L_{C} \circ\left\{\mathcal{E}_{U^{\prime}} * \mathcal{E}_{V^{\prime}}(A)\right\} \circ L_{C}^{*}$.

Proof. $\mathcal{E}_{U^{\prime}} * \mathcal{E}_{V^{\prime}}=\mathcal{E}_{U^{\prime} V^{\prime}}$ is the spectral measure associated with the unitary operator $U^{\prime} V^{\prime}$. But $U^{\prime} V^{\prime}=L_{C}^{*} U L_{C} L_{C}^{*} V L_{C}=$ $L_{C}^{*} C U V L_{C}=L_{C}^{*} U V L_{C}=V^{\prime} U^{\prime}=L_{C}^{*} V U L_{C}$. As $C$ commutes with the unitary operators $U V$ and $V U$, we can conclude that $L_{C}^{*} U V L_{C}$ and $L_{C}^{*} V U L_{C}$ are unitary operators of $\operatorname{Im} C$ which are equal, and of associated spectral measure the application $A \in \mathcal{B} \mapsto L_{C}^{*}\left(\mathcal{E}_{U V} A\right) L_{C} \in \mathcal{P}(\operatorname{Im} C)$ or equivalently $A \in \mathcal{B} \mapsto L_{C}^{*}\left(\mathcal{E}_{V U} A\right) L_{C} \in \mathcal{P}(\operatorname{Im} C)$.

As $U^{\prime} V^{\prime}=L_{C}^{*} U V L_{C}=L_{C}^{*} V U L_{C}$, we have, for any $A \in \mathcal{B}$,

$$
\mathcal{E}_{U^{\prime}} * \mathcal{E}_{V^{\prime}}(A)=L_{C}^{*} \circ\left(\mathcal{E}_{U V} A\right) \circ L_{C}=L_{C}^{*} \circ\left(\mathcal{E}_{V U} A\right) \circ L_{C}
$$

Therefore,

$$
L_{C} \circ\left\{\mathcal{E}_{U^{\prime}} * \mathcal{E}_{V^{\prime}}(A)\right\} \circ L_{C}^{*}=C\left(\mathcal{E}_{U V} A\right) C=C\left(\mathcal{E}_{V U} A\right) C=C \mathcal{E}_{U V} A=C \mathcal{E}_{V U} A .
$$

We recall that $C$ commutes with the unitary operators $U V$ and $V U$, so with the projectors $\mathcal{E}_{U V} A$ and $\mathcal{E}_{V U} A$. As $C$ commutes with $\mathcal{E}_{U V} A$ and $\mathcal{E}_{V U} A$, it commutes with $\inf \left(\mathcal{E}_{U V} A, \mathcal{E}_{V U} A\right)$ and $C \inf \left(\mathcal{E}_{U V} A, \mathcal{E}_{V U} A\right)=\inf \left(C \mathcal{E}_{U V} A, C \mathcal{E}_{V U} A\right)=$ $C \mathcal{E}_{U V} A=C \mathcal{E}_{V U} A$.

Let $A$ be an element of $\mathcal{B}$. Then

$$
L_{C}^{*} \circ\left\{\mathcal{E}_{U^{\prime}} * \mathcal{E}_{V^{\prime}}(A)\right\} \circ L_{C}^{*}=C \mathcal{E}_{U V} A=C \mathcal{E}_{V U} A=\inf \left(C \mathcal{E}_{U V} A, C \mathcal{E}_{V U} A\right)=\inf \left\{\inf \left(C, \mathcal{E}_{U V} A\right), \inf \left(C, \mathcal{E}_{V U} A\right)\right\} .
$$

However, the right-hand term is a lower bound of the family $\left\{C, \mathcal{E}_{U V} A, \mathcal{E}_{V U} A\right\}$, so

$$
\inf \left\{\inf \left(C, \mathcal{E}_{U V} A\right), \inf \left(C, \mathcal{E}_{V U} A\right)\right\} \ll \inf \left(C, \mathcal{E}_{U V} A, \mathcal{E}_{V U} A\right) .
$$

Moreover, $\inf \left(C, \mathcal{E}_{U V} A, \mathcal{E}_{V U} A\right)$ is a lower bound of the families $\left\{C, \mathcal{E}_{U V} A\right\}$ and $\left\{C, \mathcal{E}_{V U} A\right\}$, so $\inf \left(C, \mathcal{E}_{U V} A, \mathcal{E}_{V U} A\right) \ll$ $\inf \left(C, \mathcal{E}_{U V} A\right)$ and $\inf \left(C, \mathcal{E}_{U V} A, \mathcal{E}_{V U} A\right) \ll \inf \left(C, \mathcal{E}_{V U} A\right)$ and then

$$
\inf \left\{C, \mathcal{E}_{U V} A, \mathcal{E}_{V U} A\right\} \ll \inf \left\{\inf \left\{C, \mathcal{E}_{U V} A\right\}, \inf \left\{C, \mathcal{E}_{V U} A\right\}\right\},
$$

which completes the proof of claim (i). Furthermore, we have

$$
L_{C} \circ\left\{\mathcal{E}_{U^{\prime}} * \mathcal{E}_{V^{\prime}}(A)\right\} \circ L_{C}^{*}=C \mathcal{E}_{U V} A=C \mathcal{E}_{V U} A=\inf \left(C, \mathcal{E}_{U V} A, \mathcal{E}_{V U} A\right) .
$$


Let $A$ be an element of $\mathcal{B}, L_{C} \circ\left\{\mathcal{E}_{U^{\prime}} * \mathcal{E}_{V^{\prime}}(A)\right\} \circ L_{C}^{*}=C \mathcal{E}_{U V} A=C \mathcal{E}_{V U} A=\inf \left(C, \mathcal{E}_{U V} A, \mathcal{E}_{V U} A\right)$ is then a lower bound of the family $\left\{C, \mathcal{E}_{U V} A, \mathcal{E}_{V U} A\right\}$ and then of the family $\left\{\mathcal{E}_{U V} A, \mathcal{E}_{V U} A\right\}$. Thus

$$
L_{C} \circ\left\{\mathcal{E}_{U^{\prime}} * \mathcal{E}_{V^{\prime}}(A)\right\} \circ L_{C}^{*} \ll \inf \left(\mathcal{E}_{U V} A, \mathcal{E}_{V U} A\right) \ll \sup \left(\mathcal{E}_{U V} A, \mathcal{E}_{V U} A\right) .
$$

For any $A \in \mathcal{B}$, we can also write

$$
L_{C} \circ\left\{\mathcal{E}_{U^{\prime}} * \mathcal{E}_{V^{\prime}}(\complement A)\right\} \circ L_{C}^{*} \ll \inf \left\{\mathcal{E}_{U V}(\complement A), \mathcal{E}_{V U}(\complement A)\right\}
$$

and then, taking the orthogonal, $\sup \left(\mathcal{E}_{U V} A, \mathcal{E}_{V U} A\right) \ll I-L_{C} \circ\left\{\mathcal{E}_{U^{\prime}} * \mathcal{E}_{V^{\prime}}(\complement A)\right\} \circ L_{C}^{*}$, i.e.,

$$
\sup \left(\mathcal{E}_{U V} A, \mathcal{E}_{V U} A\right) \ll C^{\perp}+L_{C} L_{C}^{*}-L_{C} \circ\left\{\mathcal{E}_{U^{\prime}} * \mathcal{E}_{V^{\prime}}(\complement A)\right\} \circ L_{C}^{*} .
$$

Therefore,

$$
\sup \left(\mathcal{E}_{U V} A, \mathcal{E}_{V U} A\right) \ll C^{\perp}+L_{C} \circ\left[I_{\mathrm{Im} C}-\left\{\mathcal{E}_{U^{\prime}} * \mathcal{E}_{V^{\prime}}(\complement A)\right\}\right] \circ L_{C}^{*}=C^{\perp}+L_{C} \circ\left(\mathcal{E}_{U^{\prime}} * \mathcal{E}_{V^{\prime}} A\right) \circ L_{C}^{*} .
$$

Thus, taking into account (5), we can conclude that

$$
L_{C} \circ\left\{\mathcal{E}_{U^{\prime}} * \mathcal{E}_{V^{\prime}}(A)\right\} \circ L_{C}^{*} \ll \inf \left(\mathcal{E}_{U V} A, \mathcal{E}_{V U} A\right) \ll \sup \left(\mathcal{E}_{U V} A, \mathcal{E}_{V U} A\right) \ll C^{\perp}+L_{C} \circ\left\{\mathcal{E}_{U^{\prime}} * \mathcal{E}_{V^{\prime}}(A)\right\} \circ L_{C}^{*}
$$

for all $A \in \mathcal{B}$.

From Part (i) of Proposition 17, we deduce the following.

Corollary 4. When $A \in \mathcal{B}$, we have $\mathcal{E}_{U^{\prime}} * \mathcal{E}_{V^{\prime}}(A)=0$ if and only if $\inf \left(C, \mathcal{E}_{U V} A, \mathcal{E}_{V U} A\right)=0$.

Proof. If $0=\inf \left(C, \mathcal{E}_{U V} A, \mathcal{E}_{V U} A\right)=L_{C} \circ\left\{\mathcal{E}_{U^{\prime}} * \mathcal{E}_{V^{\prime}}(A)\right\} \circ L_{C}^{*}$, then $0=L_{C}^{*} \circ 0 \circ L_{C}=L_{C}^{*} \circ L_{C} \circ\left\{\mathcal{E}_{U^{\prime}} * \mathcal{E}_{V^{\prime}}(A)\right\} \circ L_{C}^{*} \circ L_{C}=$ $\mathcal{E}_{U^{\prime}} * \mathcal{E}_{V^{\prime}}(A)$ because $L_{C}^{*} \circ L_{C}=I_{\operatorname{Im} C}$. As for the converse, it is obvious.

As $\inf \left(C, \mathcal{E}_{U V} A, \mathcal{E}_{V U} A\right) \ll \inf \left(\mathcal{E}_{U V} A, \mathcal{E}_{V U} A\right)$, we also have the following result.

Corollary 5. If $A \in \mathcal{B}$ is such that $\inf \left(\mathcal{E}_{U V} A, \mathcal{E}_{V U} A\right)=0$, then $\mathcal{E}_{U^{\prime}} * \mathcal{E}_{V^{\prime}}(A)=0$.

This is a way to obtain a sufficient condition in order to have $\mathcal{E}_{U^{\prime}} * \mathcal{E}_{V^{\prime}}(A)=0$. Let us now examine a sufficient condition in order to have $\mathcal{E}_{U^{\prime}} * \mathcal{E}_{V^{\prime}}(A)=I$.

Corollary 6. If $A \in \mathcal{B}$ is such that $\sup \left(\mathcal{E}_{U V} A, \mathcal{E}_{V U} A\right)=I$, then $\mathcal{E}_{U^{\prime}} * \mathcal{E}_{V^{\prime}}(A)=I$.

Proof. From $I=\sup \left(\mathcal{E}_{U V} A, \mathcal{E}_{V U} A\right)$, taking the orthogonal, we have $0=\inf \left(\mathcal{E}_{U V} \complement A, \mathcal{E}_{V U} \complement A\right)$ and then, from Corollary $5, \mathcal{E}_{U^{\prime}} * \mathcal{E}_{V^{\prime}}(\complement A)=0$, i.e., $\mathcal{E}_{U^{\prime}} * \mathcal{E}_{V^{\prime}}(A)=I$.

Part (ii) of Proposition 17 gives an upper bound and a lower bound of the projectors $\inf \left(\mathcal{E}_{U V} A, \mathcal{E}_{V U} A\right)$ and $\sup \left(\mathcal{E}_{U V} A, \mathcal{E}_{V U} A\right)$. These projectors give an idea of the difference between the spectral measures $\mathcal{E}_{U V}$ and $\mathcal{E}_{V U}$. Note that the inequalities of Part (ii) of Proposition 17 are optimal in the sense that the inequalities become equalities in some particular cases: $L_{C} \circ\left\{\mathcal{E}_{U^{\prime}} * \mathcal{E}_{V^{\prime}}(\emptyset)\right\} \circ L_{C}^{*}=\inf \left\{\mathcal{E}_{U V}(\emptyset), \mathcal{E}_{V U}(\emptyset)\right\}$ and $\sup \left\{\mathcal{E}_{U V}(\Pi), \mathcal{E}_{V U}(\Pi)\right\}=C^{\perp}+L_{C} \circ\left\{\mathcal{E}_{U^{\prime}} *\right.$ $\left.\mathcal{E}_{V^{\prime}}(\Pi)\right\} \circ L_{C}^{*}$. The following result enables us to better understand the conditions for such equalities.

Proposition 18. For arbitrary $A \in \mathcal{B}$, the following assertions are equivalent.

(i) $L_{C} \circ\left\{\mathcal{E}_{U^{\prime}} * \mathcal{E}_{V^{\prime}}(A)\right\} \circ L_{C}^{*}=\inf \left(\mathcal{E}_{U V} A, \mathcal{E}_{V U} A\right)$.

(ii) $\inf \left(\mathcal{E}_{U V} A, \mathcal{E}_{V U} A\right) \ll C$.

(iii) $C^{\perp} \ll \sup \left\{\mathcal{E}_{U V}(\complement A), \mathcal{E}_{V U}(\complement A)\right\}$.

(iv) $\sup \left\{\mathcal{E}_{U V}(\complement A), \mathcal{E}_{V U}(\complement A)\right\}=C^{\perp}+L_{C} \circ\left\{\mathcal{E}_{U^{\prime}} * \mathcal{E}_{V^{\prime}}(\complement A)\right\} \circ L_{C}^{*}$. 
Proof. We will prove successively that (i) implies (ii), (ii) implies (iii), (iii) implies (iv), and (iv) implies (i).

Assume (i) holds. If $L_{C} \circ\left\{\mathcal{E}_{U^{\prime}} * \mathcal{E}_{V^{\prime}}(A)\right\} \circ L_{C}^{*}=\inf \left(\mathcal{E}_{U V} A, \mathcal{E}_{V U} A\right)$, from Proposition 17, we have $C \inf \left(\mathcal{E}_{U V} A, \mathcal{E}_{V U} A\right)=$ $\inf \left(\mathcal{E}_{U V} A, \mathcal{E}_{V U} A\right)$, i.e., $\inf \left(\mathcal{E}_{U V} A, \mathcal{E}_{V U} A\right) \ll C$, and hence (ii) holds.

We prove the second implication by considering orthogonal elements. If $C^{\perp} \ll \sup \left\{\mathcal{E}_{U V}(\complement A), \mathcal{E}_{V U}(\complement A)\right\}$, we have

$$
C^{\perp}=C^{\perp} \sup \left\{\mathcal{E}_{U V}(\complement A), \mathcal{E}_{V U}(\complement A)\right\}=\sup \left\{\mathcal{E}_{U V}(\complement A), \mathcal{E}_{V U}(\complement A)\right\}-C \sup \left\{\mathcal{E}_{U V}(\complement A), \mathcal{E}_{V U}(\complement A)\right\} .
$$

So

$$
\sup \left\{\mathcal{E}_{U V}(\complement A), \mathcal{E}_{V U}(\complement A)\right\}=C^{\perp}+C \sup \left\{\mathcal{E}_{U V}(\complement A), \mathcal{E}_{V U}(\complement A)\right\} .
$$

But, taking into account Proposition 17,

$$
\begin{aligned}
C \sup \left\{\mathcal{E}_{U V}(\complement A), \mathcal{E}_{V U}(\complement A)\right\} & =C\left[I-\inf \left\{\mathcal{E}_{U V}(A), \mathcal{E}_{V U}(A)\right\}\right]=C-C \inf \left\{\mathcal{E}_{U V}(A), \mathcal{E}_{V U}(A)\right\} \\
& =L_{C} L_{C}^{*}-L_{C}\left\{\mathcal{E}_{U^{\prime}} * \mathcal{E}_{V^{\prime}}(A)\right\} L_{C}^{*}=L_{C}\left\{I_{\operatorname{Im} C}-\mathcal{E}_{U^{\prime}} * \mathcal{E}_{V^{\prime}}(A)\right\} L_{C}^{*}=L_{C} \circ\left\{\mathcal{E}_{U^{\prime}} * \mathcal{E}_{V^{\prime}}(\complement A)\right\} L_{C}^{*} .
\end{aligned}
$$

In the light of (6), this gives

$$
\sup \left\{\mathcal{E}_{U V}(\complement A), \mathcal{E}_{V U}(\complement A)\right\}=C^{\perp}+L_{C} \circ\left\{\mathcal{E}_{U^{\prime}} * \mathcal{E}_{V^{\prime}}(\complement A)\right\} \circ L_{C}^{*},
$$

which proves the third implication.

Finally, if $\sup \left\{\mathcal{E}_{U V}(\complement A), \mathcal{E}_{V U}(\complement A)\right\}=C^{\perp}+L_{C} \circ\left\{\mathcal{E}_{U^{\prime}} * \mathcal{E}_{V^{\prime}}(\complement A)\right\} \circ L_{C}^{*}$, taking the orthogonal, we get

$$
\begin{aligned}
\inf \left\{\mathcal{E}_{U V}(A), \mathcal{E}_{V U}(A)\right\} & =C-L_{C} \circ\left\{\mathcal{E}_{U^{\prime}} * \mathcal{E}_{V^{\prime}}(\complement A)\right\} \circ L_{C}^{*}=L_{C} L_{C}^{*}-L_{C} \circ\left\{\mathcal{E}_{U^{\prime}} * \mathcal{E}_{V^{\prime}}(\complement A)\right\} \circ L_{C}^{*} \\
& =L_{C} \circ\left\{I_{\operatorname{Im} C}-\mathcal{E}_{U^{\prime}} * \mathcal{E}_{V^{\prime}}(\complement A)\right\} \circ L_{C}^{*}=L_{C} \circ\left\{\mathcal{E}_{U^{\prime}} * \mathcal{E}_{V^{\prime}}(A)\right\} \circ L_{C}^{*},
\end{aligned}
$$

which completes the proof.

Let us now examine a relation which evokes a convolution formula.

Proposition 19. For any pair $(U, V)$ of unitary operators and for any $\lambda \in \Pi$, we have $\sup \left\{\inf \left\{\mathcal{E}_{U}\left(\{\lambda\} \ominus \lambda^{\prime}\right), \mathcal{E}_{V}\left\{\lambda^{\prime}\right\}\right\}\right.$ : $\left.\lambda^{\prime} \in \Pi\right\} \ll \mathcal{E}_{U V}\{\lambda\}$.

Proof. Let $X$ be an element of $\operatorname{Im} \inf \left\{\mathcal{E}_{U}\left(\{\lambda\} \ominus \lambda^{\prime}\right), \mathcal{E}_{V}\left\{\lambda^{\prime}\right\}\right\}=\operatorname{Im} \mathcal{E}_{U}\left(\{\lambda\} \ominus \lambda^{\prime}\right) \cap \operatorname{Im} \mathcal{E}_{V}\left\{\lambda^{\prime}\right\}$. From $X \in \operatorname{Im} \mathcal{E}_{U}\left(\{\lambda\} \ominus \lambda^{\prime}\right)$, we deduce that

$$
U X=\mathrm{e}^{i\left(\lambda \ominus \lambda^{\prime}\right)} X
$$

From $X \in \operatorname{Im} \mathcal{E}_{V}\left\{\lambda^{\prime}\right\}$, we also deduce that

$$
V X=\mathrm{e}^{i \lambda^{\prime}} X
$$

Combining (7) and (8), we can write $U V X=\mathrm{e}^{i \lambda} X$, so $X \in \operatorname{Im} \mathcal{E}_{U V}\{\lambda\}$. We have just proved that $\operatorname{Im} \inf \left\{\mathcal{E}_{U}(\{\lambda\} \ominus\right.$ $\left.\left.\lambda^{\prime}\right), \mathcal{E}_{V}\left\{\lambda^{\prime}\right\}\right\} \subset \operatorname{Im} \mathcal{E}_{U V}\{\lambda\}$, i.e., $\inf \left\{\mathcal{E}_{U}\left(\{\lambda\} \ominus \lambda^{\prime}\right), \mathcal{E}_{V}\left\{\lambda^{\prime}\right\}\right\} \ll \mathcal{E}_{U V}\{\lambda\}$. The projector $\mathcal{E}_{U V}\{\lambda\}$ being an upper bound of the family $\left\{\inf \left\{\mathcal{E}_{U}\left(\{\lambda\} \ominus \lambda^{\prime}\right), \mathcal{E}_{V}\left\{\lambda^{\prime}\right\}\right\}: \lambda^{\prime} \in \Pi\right\}$, we can write $\sup \left\{\inf \left\{\mathcal{E}_{U}\left(\{\lambda\} \ominus \lambda^{\prime}\right), \mathcal{E}_{V}\left\{\lambda^{\prime}\right\}\right\}: \lambda^{\prime} \in \Pi\right\} \ll \mathcal{E}_{U V}\{\lambda\}$, and the proposition is proved.

Remark 7. (a) An upper bound of projectors can be a sum, e.g., if the projectors $P_{1}$ and $P_{2}$ commute, then $\sup \left(P_{1}, P_{2}\right)=P_{1}+P_{2}$. Then the first term of the inequality of the proposition becomes similar to the expression of a convolution product.

(b) In some particular cases, the formula may become $\sup \left\{\inf \left\{\mathcal{E}_{U}\left(\{\lambda\} \ominus \lambda^{\prime}\right), \mathcal{E}_{V}\left\{\lambda^{\prime}\right\}\right\}: \lambda^{\prime} \in \Pi\right\}=0 \ll \mathcal{E}_{U V}\{\lambda\}$, and in other cases, it becomes $\sup \left\{\inf \left\{\mathcal{E}_{U}\left(\{\lambda\} \ominus \lambda^{\prime}\right), \mathcal{E}_{V}\left\{\lambda^{\prime}\right\}\right\}: \lambda^{\prime} \in \Pi\right\}=\mathcal{E}_{U V}\{\lambda\}$.

We conclude this section with a final relation between the spectral measures $\mathcal{E}_{U V}$ and $\mathcal{E}_{V U}$. The unitary operators $U V$ and $V U$ are unitary equivalent: $U^{-1}(U V) U=V U$. We are going to see that this can be transposed to the spectral measures $\mathcal{E}_{U V}$ and $\mathcal{E}_{V U}$.

Proposition 20. (i) For any $A \in \mathcal{B}, \alpha A=U^{-1}\left(\mathcal{E}_{U V} A\right) U$ is a projector. (ii) the application $\alpha: A \in \mathcal{B} \mapsto$ $U^{-1}\left(\mathcal{E}_{U V} A\right) U \in \mathcal{P}(H)$ is a spectral measure. (iii) For any $A \in \mathcal{B}, \mathcal{E}_{V U} A=U^{-1}\left(\mathcal{E}_{U V} A\right) U$. 
Proof. It is easy to check the first two parts. Now denote by $W$ the unitary operator of associated spectral measure $\alpha$. For a given $X$ of $H$, we have $W X=\int e^{i \cdot 1} \mathrm{~d} Z_{\alpha}^{X}$. But $Z_{\alpha}^{X}=U^{-1} \circ Z_{\mathcal{E}_{U V}}^{U X}$, because for any $A \in \mathcal{B}$,

$$
Z_{\alpha}^{X} A=(\alpha A) X=U^{-1}\left(\mathcal{E}_{U V} A\right) U X=U^{-1} \circ Z_{\mathcal{E}_{U V}}^{U X} A .
$$

As a result

$$
W X=\int e^{i \cdot 1} \mathrm{~d} U^{-1} \circ Z_{\mathcal{E}_{U V} X}^{U X}=U^{-1} \int e^{i \cdot 1} \mathrm{~d} Z_{\mathcal{E}_{U V}}^{U X}=U^{-1} U V U X,
$$

because $\mathcal{E}_{U V}$ is the spectral measure associated with $U V$. The relation $W X=U^{-1} U V U X$, for any $X \in H$, allows us to write $W=U^{-1} \circ U V \circ U$. This means that $U^{-1} \circ U V \circ U$, i.e., $V U$, has $\alpha$ as associated spectral measure. And then, for any $A \in \mathcal{B}, \mathcal{E}_{V U} A=\alpha A=U^{-1}\left(\mathcal{E}_{U V} A\right) U$.

\section{The finite spectrum case and convergence properties}

In this section, we study the structure of the commuters $C_{P, U}$ and $C_{U, V}$ when the spectral measures which are associated with the unitary operators $U$ and $V$ are concentrated on a finite number of elements of $\Pi$. This will allow us to specify the nature of the projectors $C_{P, U}$ and $C_{U, V}$. Then, when this assumption no longer holds, we will establish properties of $r$-convergence of the form: $C_{P, U}=\lim _{n}^{r} C_{P, U_{n}}$ and $C_{U, V}=\lim _{n}^{r} C_{U_{n}, V_{n}}$, where $U_{n}$ and $V_{n}$ are unitary operators, respectively defined from $U$ and $V$, thanks to a partition of $\Pi$ for which the associated spectral measures are concentrated on a finite number of elements of $\Pi$.

To this end, we first give some reminders and specific notation for this section. For any $n \in \mathbb{N}$, set $A_{n, k}=$ $\left[-\pi+k 2 \pi / 2^{n},-\pi+(k+1) 2 \pi / 2^{n}\right)$ for all $k \in\left\{0, \ldots, 2^{n}-1\right\}$. It is clear that $\left\{A_{n, k}: k=0, \ldots, 2^{n}-1\right\}$ is a family of elements of $\mathcal{B}$ which constitutes a partition of $\Pi$. The application $\mathcal{L}_{n}=\sum_{k=0}^{2^{n}-1}\left(-\pi+k 2 \pi / 2^{n}\right) 1_{A_{n, k}}$ from $\Pi$ into itself is measurable and such that $\mathcal{L}_{n} \circ \mathcal{L}_{n+1}=\mathcal{L}_{n}$. If $U$ is a unitary operator and $U_{n}$ the unitary operator of associated spectral measure $\mathcal{L}_{n} \mathcal{E}_{U}\left(\mathcal{E}_{U_{n}}=\mathcal{L}_{n} \mathcal{E}_{U}\right)$, then

$$
\mathcal{L}_{n} \mathcal{E}_{U}=\sum_{k=0}^{2^{n}-1} \delta_{-\pi+k 2 \pi / 2^{n}} \mathcal{E}_{U} A_{n, k}, \quad U_{n}=\sum_{k=0}^{2^{n}-1} e^{i\left(-\pi+k 2 \pi / 2^{n}\right)} \mathcal{E}_{U} A_{n, k}, \quad U X=\lim _{n} \sum_{k=0}^{2^{n}-1} e^{i\left(-\pi+k 2 \pi / 2^{n}\right)}\left(\mathcal{E}_{U} A_{n, k}\right) X=\lim _{n} U_{n} X .
$$

We first examine the expression of $C_{U, V}$ when the spectra of $U$ and $V$ are finite.

Proposition 21. Let $\left\{P_{j}: j \in J\right\}$ and $\left\{D_{\ell}: \ell \in L\right\}$ be two finite families of projectors such that $I=\sum_{j \in J} P_{j}=\sum_{\ell \in L} D_{l}$ and such that $P_{j} P_{j^{\prime}}=D_{\ell} D_{\ell^{\prime}}=0$, for any pairs $\left(j, j^{\prime}\right)$ and $\left(\ell, \ell^{\prime}\right)$ of distinct elements of $J$ and L, respectively. If $\left\{\lambda_{j}: j \in J\right\}$ and $\left\{\mu_{\ell}: \ell \in L\right\}$ are families of distinct elements of $\Pi$, then $(i) U=\sum_{j \in J} e^{i \lambda_{j}} P_{j}$ and $V=\sum_{\ell \in L} e^{i \mu_{\ell}} D_{\ell}$ are unitary operators of $H$; (ii) $C_{U, V}=\sum_{j \in J} \sum_{\ell \in L} \inf \left(P_{j}, D_{\ell}\right)$.

Proof. Let $C=\sum_{j \in J} \sum_{\ell \in L} \inf \left(P_{j}, D_{\ell}\right)$. For any pair $\left((j, \ell),\left(j^{\prime}, \ell^{\prime}\right)\right)$ of distinct elements of $J \times L$, we have $\inf \left(P_{j}, D_{\ell}\right) \circ$ $\inf \left(P_{j^{\prime}}, D_{\ell^{\prime}}\right)=0$, so $C$ is a projector. It is easy to verify that $U C=C U$, that $V C=C V$ and that $V C U=U C V$, so that $C$ is a commuter of the unitary operators $U$ and $V$. Therefore,

$$
C \ll C_{U, V} .
$$

Let $X$ be an element of $\operatorname{Im} C_{U, V}=\cap_{(A, B) \in \mathcal{B} \times \mathcal{B}} \operatorname{Ker}\left\{\left(\mathcal{E}_{U} A\right)\left(\mathcal{E}_{V} B\right)-\left(\mathcal{E}_{V} B\right)\left(\mathcal{E}_{U} A\right)\right\}$. For any $(A, B) \in \mathcal{B} \times \mathcal{B}$, we have $\left(\mathcal{E}_{U} A\right)\left(\mathcal{E}_{V} B\right) X=\left(\mathcal{E}_{V} B\right)\left(\mathcal{E}_{U} A\right) X$. Let $(j, \ell)$ be an element of $J \times L$. As $\left(\left\{\lambda_{j}\right\},\left\{\mu_{\ell}\right\}\right)$ is an element of $\mathcal{B} \times \mathcal{B}$, we can write

$$
P_{j} D_{\ell} X=\left(\mathcal{E}_{U}\left\{\lambda_{j}\right\}\right)\left(\mathcal{E}_{V}\left\{\mu_{\ell}\right\}\right) X=\left(\mathcal{E}_{V}\left\{\mu_{\ell}\right\}\right)\left(\mathcal{E}_{U}\left\{\lambda_{j}\right\}\right) X=D_{\ell} P_{j} X
$$

So as $P_{j} D_{\ell} X=D_{\ell} P_{j} X \in \operatorname{Im} P_{j} \cap \operatorname{Im} D_{\ell}=\operatorname{Im} \inf \left(P_{j}, D_{\ell}\right)$, we have $P_{j} D_{\ell} X=\inf \left(P_{j}, D_{\ell}\right) P_{j} D_{\ell} X=\inf \left(P_{j}, D_{\ell}\right) X$. As this holds for all $(j, \ell) \in J \times L$, we deduce that

$$
C X=\sum_{j \in J} \sum_{\ell \in L} \inf \left(P_{j}, D_{\ell}\right) X=\sum_{j \in J} \sum_{\ell \in L} P_{j} D_{\ell} X=\sum_{j \in J} P_{j} X=X
$$

We have just proved that $\operatorname{Im} C_{U, V} \subset \operatorname{Im} C$, so that $C_{U, V} \ll C$, which, combined with (9), gives $C_{U, V}=C=$ $\sum_{j \in J} \sum_{\ell \in L} \inf \left(P_{j}, D_{\ell}\right)$. 
Remark 8. If $P$ is a projector, as $e^{i P}=e^{i} P+P^{\perp}$, so as $\mathcal{E}_{e^{i P}}=\delta_{0} P^{\perp}+\delta_{1} P$, we have

$$
C_{P, V}=C_{e^{i P}, V}=\sum_{\ell \in L} \inf \left(P^{\perp}, D_{\ell}\right)+\sum_{\ell \in L} \inf \left(P, D_{\ell}\right)=\sum_{\ell \in L}\left\{\inf \left(P, D_{\ell}\right)+\inf \left(P^{\perp}, D_{\ell}\right)\right\} .
$$

We now state a final preliminary result before the major result of this section.

Lemma 11. Let $\mathcal{L}$ be a measurable application from $\Pi$ into itself, $U$ and $V$ be two unitary operators, and $U^{\prime}$ and $V^{\prime}$ the unitary operators of the corresponding spectral measures $\mathcal{L}\left(\mathcal{E}_{U}\right)$ and $\mathcal{L}\left(\mathcal{E}_{V}\right)$. Then we have $C_{U, V} \ll C_{U^{\prime}, V^{\prime}}$.

Proof. As $\left\{C_{\mathcal{E}_{U^{\prime}} A, \mathcal{E}_{V^{\prime}} B}:(A, B) \in \mathcal{B} \times \mathcal{B}\right\}=\left\{C_{\mathcal{E}_{U} \mathcal{L}^{-1} A, \mathcal{E}_{V} \mathcal{L}^{-1} B}:(A, B) \in \mathcal{B} \times \mathcal{B}\right\} \subset\left\{C_{\mathcal{E}_{U} A, \mathcal{E}_{V} B}:(A, B) \in \mathcal{B} \times \mathcal{B}\right\}$, we can write $C_{U, V}=\inf \left\{C_{\mathcal{E}_{U} A, \mathcal{E}_{V} B}:(A, B) \in \mathcal{B} \times \mathcal{B}\right\} \ll \inf \left\{C_{\mathcal{E}_{U^{\prime}} A, \mathcal{E}_{V^{\prime}} B}:(A, B) \in \mathcal{B} \times \mathcal{B}\right\}=C_{U^{\prime}, V^{\prime}}$.

Here are some consequences of these results.

Proposition 22. If $(U, V)$ is a pair of unitary operators, then $\left(\sum_{k=0}^{2^{n}-1} \sum_{\ell=0}^{2^{n}-1} \inf \left(\mathcal{E}_{U} A_{n, k}, \mathcal{E}_{V} A_{n, \ell}\right), n \in \mathbb{N}\right)$ is a decreasing sequence of projectors which converges to $C_{U, V}$.

Proof. For any $n \in \mathbb{N}$, denote by $U_{n}$ and $V_{n}$ the unitary operators of respective associated spectral measures $\mathcal{L}_{n} \mathcal{E}_{U}$ and $\mathcal{L}_{n} \mathcal{E}_{V}$. Then we have $\mathcal{E}_{U_{n}}=\mathcal{L}_{n} \mathcal{E}_{U}$ and $\mathcal{E}_{V_{n}}=\mathcal{L}_{n} \mathcal{E}_{V}$. So $\mathcal{E}_{U_{n}}=\mathcal{L}_{n} \mathcal{L}_{n+1} \mathcal{E}_{U}=\mathcal{L}_{n} \mathcal{E}_{U_{n+1}}$ and $\mathcal{E}_{V_{n}}=\mathcal{L}_{n} \mathcal{E}_{V_{n+1}}$. From Lemma 11, we deduce that $C_{U_{n+1}, V_{n+1}} \ll C_{U_{n}, V_{n}}$. So $\left(C_{U_{n}, V_{n}}, n \in \mathbb{N}\right)$ is a decreasing sequence of projectors. From Section 2, it is $r$-convergent, and $\lim _{n}^{r} C_{U_{n}, V_{n}}=\inf \left(C_{U_{n}, V_{n}}, n \in \mathbb{N}\right)$. Let $C=\lim _{n}^{r} C_{U_{n}, V_{n}}$. Still from Lemma 11, we have $C_{U, V} \ll C_{U_{n}, V_{n}}$ for all $n \in \mathbb{N}$. Therefore,

$$
C_{U, V} \ll C
$$

The projector $C$ commutes with $U$ because

$$
\begin{aligned}
\|U C X-C U X\| & \leq\left\|U C X-U_{n} C X\right\|+\left\|U_{n} C X-U_{n} C_{U_{n}, V_{n}} X\right\|+\left\|C_{U_{n}, V_{n}} U_{n} X-C_{U_{n}, V_{n}} U X\right\|+\left\|C_{U_{n}, V_{n}} U X-C U X\right\| \\
& \leq\left\|U C X-U_{n} C X\right\|+\left\|C X-C_{U_{n}, V_{n}} X\right\|+\left\|U_{n} X-U X\right\|+\left\|C_{U_{n}, V_{n}} U X-C U X\right\|,
\end{aligned}
$$

but

$$
\lim _{n \rightarrow \infty}\left\|U C X-U_{n} C X\right\|=\lim _{n \rightarrow \infty}\left\|C X-C_{U_{n}, V_{n}} X\right\|=\lim _{n \rightarrow \infty}\left\|U_{n} X-U X\right\|=\lim _{n \rightarrow \infty}\left\|C_{U_{n}, V_{n}} U X-C U X\right\|=0 .
$$

Hence $\|U C X-C U X\|=0$ for every $X \in H$. That is, $C$ and $U$ commute. In the same way, we can prove that $V C=C V$.

For any $X \in H$, we also can write

$$
\begin{aligned}
\|U C V X-V C U X\| & \leq\left\|U C V X-U_{n} C V X\right\|+\left\|U_{n} C V X-U_{n} C_{U_{n}, V_{n}} V X\right\|+\left\|U_{n} C_{U_{n}, V_{n}} V X-U_{n} C_{U_{n}, V_{n}} V_{n} X\right\| \\
& +\left\|V_{n} C_{U_{n}, V_{n}} U_{n} X-V_{n} C_{U_{n}, V_{n}} U X\right\|+\left\|V_{n} C_{U_{n}, V_{n}} U X-V_{n} C U X\right\|+\left\|V_{n} C U X-V C U X\right\| \\
& \ll\left\|U C V X-U_{n} C V X\right\|+\left\|C V X-C_{U_{n}, V_{n}} V X\right\|+\left\|V X-V_{n} X\right\| \\
& +\left\|U_{n} X-U X\right\|+\left\|C_{U_{n}, V_{n}} U X-C U X\right\|+\left\|V_{n} C U X-V C U X\right\|,
\end{aligned}
$$

but

$$
\begin{aligned}
\lim _{n \rightarrow \infty}\left\|U C V X-U_{n} C V X\right\| & =\lim _{n \rightarrow \infty}\left\|C V X-C_{U_{n}, V_{n}} V X\right\|=\lim _{n \rightarrow \infty}\left\|V_{n} X-V X\right\| \\
& =\lim _{n \rightarrow \infty}\left\|U_{n} X-U X\right\|=\lim _{n \rightarrow \infty}\left\|C_{U_{n}, V_{n}} U X-C U X\right\|=\lim _{n \rightarrow \infty}\left\|V_{n} C U X-V C U X\right\|=0 .
\end{aligned}
$$

So $\|U C V X-V C U X\|=0$, i.e., $U C V X=V C U X$ for any $X \in H$. Hence $U C V=V C U$, and we can say that $C$ is a commuter of $U$ and $V$. Then $C \ll C_{U, V}$, which, combined with (10), implies that $C_{U, V}=C=\inf \left\{C_{U_{n}, V_{n}}: n \in \mathbb{N}\right\}$. As

$$
U_{n}=\sum_{k=0}^{2^{n}-1} e^{i\left(-\pi+k 2 \pi / 2^{n}\right)} \mathcal{E}_{U} A_{n, k}, \quad V_{n}=\sum_{\ell=0}^{2^{n}-1} e^{i\left(-\pi+\ell 2 \pi / 2^{n}\right)} \mathcal{E}_{V} A_{n, \ell},
$$

it follows from Proposition 21 that

$$
C_{U_{n}, V_{n}}=\sum_{k=0}^{2^{n}-1} \sum_{\ell=0}^{2^{n}-1} \inf \left(\mathcal{E}_{U} A_{n, k}, \mathcal{E}_{V} A_{n, \ell}\right)
$$

So we can conclude that $\left\{\sum_{k=0}^{2^{n}-1} \sum_{\ell=0}^{2^{n}-1} \inf \left(\mathcal{E}_{U} A_{n, k}, \mathcal{E}_{V} A_{n, \ell}\right), n \in \mathbb{N}\right\}$ is a sequence of projectors which decreasingly converges to $C_{U, V}$. 
Remark 9. When $P$ is a projector, as $e^{i P}$ is a unitary operator such that $C_{P, V}=C_{e^{i P}, V}$, Proposition 22 implies that $\left[\sum_{\ell=0}^{2^{n}-1}\left\{\inf \left(P, \mathcal{E}_{V} A_{n, \ell}\right)+\inf \left(P^{\perp}, \mathcal{E}_{V} A_{n, \ell}\right)\right\}, n \in \mathbb{N}\right]$ is a sequence of projectors which $r$-converges to $C_{P, V}$ because, for every integer $n \geq 3$,

$$
\sum_{\ell=0}^{2^{n}-1}\left\{\inf \left(P, \mathcal{E}_{V} A_{n, \ell}\right)+\inf \left(P^{\perp}, \mathcal{E}_{V} A_{n, \ell}\right)\right\}=\sum_{k=0}^{2^{n}-1} \sum_{\ell=0}^{2^{n}-1} \inf \left(\mathcal{E}_{e^{i P}} A_{n, k}, \mathcal{E}_{V} A_{n, \ell}\right)
$$

\section{Application to the characterization of a class of families of stationary correlated series}

Let $\left(X_{n}, n \in \mathbb{Z}\right)$ be a stationary series. We propose to characterize all the stationary series $\left(X_{n}^{\prime}, n \in \mathbb{Z}\right)$, stationarily correlated with $\left(X_{n}, n \in \mathbb{Z}\right)$, and such that $\left(X_{n}^{\prime}, n \in \mathbb{Z}\right) \subset \operatorname{Im} P$, where $P$ is a projector. Of course, if $H=L^{2}(\Omega, \mathcal{A}, Q)$, Im $P$ can be of $L^{2}\left(\Omega, \mathcal{A}^{\prime}, Q\right)$ type, where $\mathcal{A}^{\prime}$ is a sub- $\sigma$-field of $\mathcal{A}$. A series solution is a series of $\mathcal{A}^{\prime}$-measurable random variables. In order to solve this problem, we need some preliminary results.

Lemma 12. If $V$ is a unitary operator of $\operatorname{Im} K, K$ being a projector of $H$, then $U=K^{\perp}+L_{K} V L_{K}^{*}$ is a unitary operator of $H$ such that $U^{n}=K^{\perp}+L_{K} V^{n} L_{K}^{*}$ for all $n \in \mathbb{Z}$.

Proof. It is easily seen that $U=K^{\perp}+L_{K} V L_{K}^{*}$ is a unitary operator. It can then be shown by induction that, for all $n \in \mathbb{N}, U^{n}=K^{\perp}+L_{K} V^{n} L_{K}^{*}$. This property extends to $\mathbb{Z}$. Indeed, when $n<0$, we have $U^{|n|}=K^{\perp}+L_{K} V^{|n|} L_{K}^{*}$, and considering the adjoint, $U^{-|n|}=K^{\perp}+L_{K} V^{-|n|} L_{K}^{*}$, i.e., $U^{n}=K^{\perp}+L_{K} V^{n} L_{K}^{*}$.

Lemma 13. If $\left(X_{n}^{1}, n \in \mathbb{N}\right)$ and $\left(X_{n}^{2}, n \in \mathbb{N}\right)$ are two stationary series that are stationarily correlated, then there exists a unitary operator $U$ of $H$ such that, for all $n \in \mathbb{Z}, U^{n} X_{0}^{1}=X_{n}^{1}$ and $U^{n} X_{0}^{2}=X_{n}^{2}$.

Proof. For any pair $\left((i, n),\left(i^{\prime}, n^{\prime}\right)\right)$ of elements of $\{1,2\} \times \mathbb{Z}$, we have $<X_{n}^{i}, X_{n^{\prime}}^{i^{\prime}}>=<X_{n+1}^{i}, X_{n^{\prime}+1}^{i^{\prime}}>$, so from [1], there exists an isometry $V$ from $\overline{\operatorname{vect}}\left\{X_{n}^{i}:(i, n) \in\{1,2\} \times \mathbb{Z}\right\}$ onto $\overline{\operatorname{vect}}\left\{X_{n+1}^{i}:(i, n) \in\{1,2\} \times \mathbb{Z}\right\}$ such that $V X_{n}^{i}=X_{n+1}^{i}$, for any $(i, n) \in\{1,2\} \times \mathbb{Z}$. Noting that $\overline{\operatorname{vect}}\left\{X_{n}^{i}:(i, n) \in\{1,2\} \times \mathbb{Z}\right\}=\overline{\operatorname{vect}}\left\{X_{n+1}^{i}:(i, n) \in\{1,2\} \times \mathbb{Z}\right\}$, we can then conclude that there exists a unitary operator $V$ of $H^{\prime}=\overline{\operatorname{vect}}\left\{X_{n}^{i}:(i, n) \in\{1,2\} \times \mathbb{Z}\right\}$ such that $V X_{n}^{i}=X_{n+1}^{i}$, for all $(i, n) \in\{1,2\} \times \mathbb{Z}$. Then, considering an element $(i, n)$ of $\{1,2\} \times \mathbb{Z}$, it is easy to check by induction that for all $n \in \mathbb{N}$, $V^{n} X_{m}^{i}=X_{n+m}^{i}$. More precisely, $V^{n} X_{m}^{i}=X_{n+m}^{i}$ for all $(i, n, m) \in\{1,2\} \times \mathbb{Z} \times \mathbb{N}$.

Let $n \in \mathbb{Z}$. If $n$ is positive and $m=0$, what precedes implies $V^{n} X_{0}^{i}=X_{n}^{i}$. If $n$ is negative, as $(i,-|n|,|n|) \in$ $\{1,2\} \times \mathbb{Z} \times \mathbb{N}$, we have $V^{|n|} X_{-|n|}^{i}=X_{|n|-|n|}^{i}$, i.e., $V^{-n} X_{n}^{i}=X_{0}^{i}$. So $X_{n}^{i}=V^{n} X_{0}^{i}$. We have just proved that $V^{n} X_{0}^{i}=X_{n}^{i}$ for all $(i, n) \in\{1,2\} \times \mathbb{Z}$.

From the previous lemma, if we denote by $K$ the projector on $H^{\prime}$, we can assert that $U=K^{\perp}+L_{K} V L_{K}^{*}$ is a unitary operator of $H$ such that $U^{n}=K^{\perp}+L_{K} V^{n} L_{K}^{*}$, for any $n \in \mathbb{Z}$. For any $(i, n) \in\{1,2\} \times \mathbb{Z}$, we can then write

$$
U^{n} X_{0}^{i}=K^{\perp} X_{0}^{i}+L_{K} V^{n} L_{K}^{*} X_{0}^{i}=0+L_{K} V^{n} X_{0}^{i}=L_{K} X_{n}^{i}=X_{n}^{i}
$$

which completes the proof.

We now examine the notion of unitary operator compatible with a stationary series.

Definition 3. We say that a unitary operator $U$ of $H$ is compatible with a stationary series $\left(X_{n}, n \in \mathbb{Z}\right)$ if $U^{n} X_{0}=X_{n}$ for all $n \in \mathbb{Z}$.

Of course, the stationary series $\left(X_{n}, n \in \mathbb{Z}\right)$ is stationarily correlated with itself, and Lemma 13 allows us to express the following.

Proposition 23. If $\left(X_{n}, n \in \mathbb{Z}\right)$ is a stationary series, then there exists at least one unitary operator of $H$ which is compatible with it.

We now have the tools we need in order to solve the problem stated at the beginning of this section.

Definition 4. Let $\left(X_{n}, n \in \mathbb{Z}\right)$ be a stationary series and $P$ be a projector of $H$. A series solution is any stationary series $\left(X_{n}^{\prime}, n \in \mathbb{Z}\right)$, stationarily correlated with $\left(X_{n}, n \in \mathbb{Z}\right)$, and such that $\left(X_{n}^{\prime}, n \in \mathbb{Z}\right) \subset \operatorname{Im} P$. 
Then we have the following property.

Proposition 24. Let $\left(X_{n}, n \in \mathbb{Z}\right)$ be a stationary series and $P$ be a projector of $H$. If $U$ is a unitary operator compatible with $\left(X_{n}, n \in \mathbb{Z}\right)$, for any $X \in H$, then the series $\left(U^{n} \inf \left(P, C_{P, U}\right) X, n \in \mathbb{Z}\right)$ is a solution. Conversely, any solution is of this type.

Proof. Let $U$ be a unitary operator, compatible with the stationary series $\left(X_{n}, n \in \mathbb{Z}\right)$. The series $\left\{U^{n} \inf \left(P, C_{P, U}\right) X, n \in\right.$ $\mathbb{Z}\}$ is stationary, stationarily correlated with $\left\{U^{n} X_{0}, n \in \mathbb{Z}\right\}$, i.e., with $\left(X_{n}, n \in \mathbb{Z}\right)$ because $\left(U^{n} X_{0}, n \in \mathbb{Z}\right)=\left(X_{n}, n \in \mathbb{Z}\right)$. Moreover, for any $n \in \mathbb{Z}$, we have

$$
U^{n} \inf \left(P, C_{P, U}\right) X=U^{n} C_{P, U} P \inf \left(P, C_{P, U}\right) X .
$$

As $C_{P, U}$ is a commuter of $P$ and $U$, it is a commuter of $P$ and $U^{n}\left(C_{P, U}=C_{\mathrm{e}^{i P}, U}\right.$ is a commuter of the unitary operators $\mathrm{e}^{i P}$ and $U$, so of the unitary operators $\mathrm{e}^{i P}$ and $U^{n}$, and then of $P$ and $U^{n}$ ), so (11) can be completed by

$$
U^{n} \inf \left(P, C_{P, U}\right) X=P C_{P, U} U^{n} \inf \left(P, C_{P, U}\right) X \text {. }
$$

We have then $\left\{U^{n} \inf \left(P, C_{P, U}\right) X, n \in \mathbb{Z}\right\} \subset \operatorname{Im} P$, which allows us to conclude that $\left\{U^{n} \inf \left(P, C_{P, U}\right) X, n \in \mathbb{Z}\right\}$ is a series solution.

Conversely, let $\left(X_{n}^{\prime}, n \in \mathbb{Z}\right)$ be a series solution. As the stationary series $\left(X_{n}, n \in \mathbb{Z}\right)$ and $\left(X_{n}^{\prime}, n \in \mathbb{Z}\right)$ are stationarily correlated, from Lemma 13 , there exists a unitary operator $U$ such that $\left(U^{n} X_{0}, n \in \mathbb{Z}\right)=\left(X_{n}, n \in \mathbb{Z}\right)$ and such that $\left(U^{n} X_{0}^{\prime}, n \in \mathbb{Z}\right)=\left(X_{n}^{\prime}, n \in \mathbb{Z}\right)$. The equality $\left(U^{n} X_{0}, n \in \mathbb{Z}\right)=\left(X_{n}, n \in \mathbb{Z}\right)$ means that $U$ is compatible with $\left(X_{n}, n \in \mathbb{Z}\right)$; see Definition 3. As $\left(X_{n}^{\prime}, n \in \mathbb{Z}\right) \subset \operatorname{Im} P$, for any $n \in \mathbb{Z}$, we can write $P U^{n} X_{0}^{\prime}=P X_{n}^{\prime}=X_{n}^{\prime}=U^{n} X_{0}^{\prime}=U^{n} P X_{0}^{\prime}$ so $X_{0}^{\prime}$ belongs to $\operatorname{Ker}\left(P U^{n}-U^{n} P\right)$. This being exact for any $n \in \mathbb{Z}, X_{0}^{\prime}$ belongs to $\cap_{n \in \mathbb{Z}} \operatorname{Ker}\left(P U^{n}-U^{n} P\right)=\operatorname{Im} C_{P, U}$, and so $X_{0}^{\prime}=C_{P, U} X_{0}^{\prime}$. Moreover, $\inf \left(P, C_{P, U}\right) X_{0}^{\prime}=P C_{P, U} X_{0}^{\prime}=P X_{0}^{\prime}=X_{0}^{\prime}$. We deduce that $X_{n}^{\prime}=U^{n} X_{0}^{\prime}=U^{n} \inf \left(P, C_{P, U}\right) X_{0}^{\prime}$. It is then clear that the series $\left(X_{n}^{\prime}, n \in \mathbb{Z}\right)$ can be written as $\left(U^{n} \inf \left(P, C_{P, U}\right) X_{0}^{\prime}, n \in \mathbb{Z}\right)$, which concludes the proof.

\section{Graphical illustrations}

As an illustration, we consider two series $\left(X_{n}, n \in \mathbb{Z}\right)$ and $\left(Y_{n}, n \in \mathbb{Z}\right)$, where $X_{n}=U^{n} X_{0}$ and $Y_{n}=V^{n} X_{0}, U$ and $V$ being two unitary operators expressed as follows

$$
U=e^{i \lambda} P+e^{i \lambda^{\prime}} P_{1}+e^{i \lambda^{\prime \prime}} P_{2}, \quad V=e^{i \lambda} P+e^{i \lambda^{\prime}} D_{1}+e^{i \lambda^{\prime \prime}} D_{2},
$$

where $P, P_{1}, P_{2}, D_{1}$ and $D_{2}$ are projectors of $H=\mathbb{C}^{k}$ such that $P_{1} D_{1} \neq 0, P+P_{1}+P_{2}=P+D_{1}+D_{2}=I$, $P P_{1}=P P_{2}=P_{1} P_{2}=P D_{1}=P D_{2}=D_{1} D_{2}=0$, and $\lambda, \lambda^{\prime}$ and $\lambda^{\prime \prime}$ are values of $\Pi$. Then, for any $n, m \in \mathbb{Z}, U^{n}=$ $e^{i \lambda n} P+e^{i \lambda^{\prime} n} P_{1}+e^{i \lambda^{\prime \prime} n} P_{2}, V^{m}=e^{i \lambda m} P+e^{i \lambda^{\prime} m} D_{1}+e^{i \lambda^{\prime \prime} m} D_{2}$ and $U^{n} V^{m}-V^{m} U^{n}=\left(e^{i \lambda^{\prime} n}-e^{i \lambda^{\prime \prime} n}\right)\left(e^{i \lambda^{\prime} m}-e^{i \lambda^{\prime \prime} m}\right)\left(P_{1} D_{1}-D_{1} P_{1}\right)$. From the latter equality, we deduce that $C_{U, V}=C_{P_{1}, D_{1}}$.

For illustration purposes, we took $H=\mathbb{C}^{5}, \lambda=\sqrt{2} / 2, \lambda^{\prime}=\sqrt{3}$ and $\lambda^{\prime \prime}=-\sqrt{5} / 5$. The fact that $\lambda, \lambda^{\prime}$ and $\lambda^{\prime \prime}$ are not elements of $2 \pi \mathbb{Q}$ guaranties the non-periodicity of the series $\left(X_{n}, n \in \mathbb{Z}\right)$ and $\left(Y_{n}, n \in \mathbb{Z}\right)$. We choose $P_{1}=a \otimes a$ and $D_{1}=b \otimes b$, where $a$ and $b$ are two linearly independent and non orthogonal normed elements of $H$. In this case, $\operatorname{Im} C_{U, V}=\{\operatorname{vect}(a, b)\}^{\perp}$.

Let $X_{0}=\varepsilon_{0} X_{0}^{0}+\varepsilon_{1} X_{0}^{1}$ be an element of $H$ such that $X_{0}^{0} \in \operatorname{Im} C_{U, V}$ and $X_{0}^{1} \in \operatorname{Im} C_{U, V}^{\perp}$. If $\varepsilon_{1}=0$, then $X_{0} \in \operatorname{Im} C_{U, V}$, and $\left(\left(U^{n} V^{n}\right) X_{0}, n \in \mathbb{Z}\right)=\left(\left(V^{n} U^{n}\right) X_{0}, n \in \mathbb{Z}\right)$. If $\varepsilon_{1}$ is small (resp. large), we say that $X_{0}$ is close to (resp. far from) $\operatorname{Im} C_{U, V}$. We generate many curves for each case by adding a random normal value to each time value and each curve.

Figure 1 shows that when $X_{0}$ is of high coefficient in $\operatorname{Im} C_{U, V}$, the two sets of curves $\left\{\left(U^{n} V^{n}\right) X_{0}: n \in \mathbb{Z}\right\}$ and $\left\{\left(V^{n} U^{n}\right) X_{0}: n \in \mathbb{Z}\right\}$ have very close shapes. When $X_{0}$ is close to $\operatorname{Im} C_{U, V}^{\perp}$, then the two sets of curves have different shapes. With the same notation, for any $X \in H$, we have

$$
\left\|U^{n} V^{m} X-V^{m} U^{n} X\right\|=\left|e^{i \lambda n}-e^{i \lambda^{\prime} n}\right| \times\left|e^{i \lambda m}-e^{i \lambda^{\prime} m}\right| \times\|<b, a><X, b>a-<a, b><X, a>b\| .
$$

The proximity between the series $\left(\left(U^{n} V^{n}\right) X_{0}, n \in \mathbb{Z}\right)$ and $\left(\left(V^{n} U^{n}\right) X_{0}, n \in \mathbb{Z}\right)$ can also be illustrated by the norms of the differences $\left\|U^{n} V^{m} X_{0}-V^{m} U^{n} X_{0}\right\|$, when $n$ and $m$ vary. In Figure 2, we see that, even though this norm varies, it becomes smaller when $X_{0}$ gets closer to $\operatorname{Im} C_{U, V}$. 

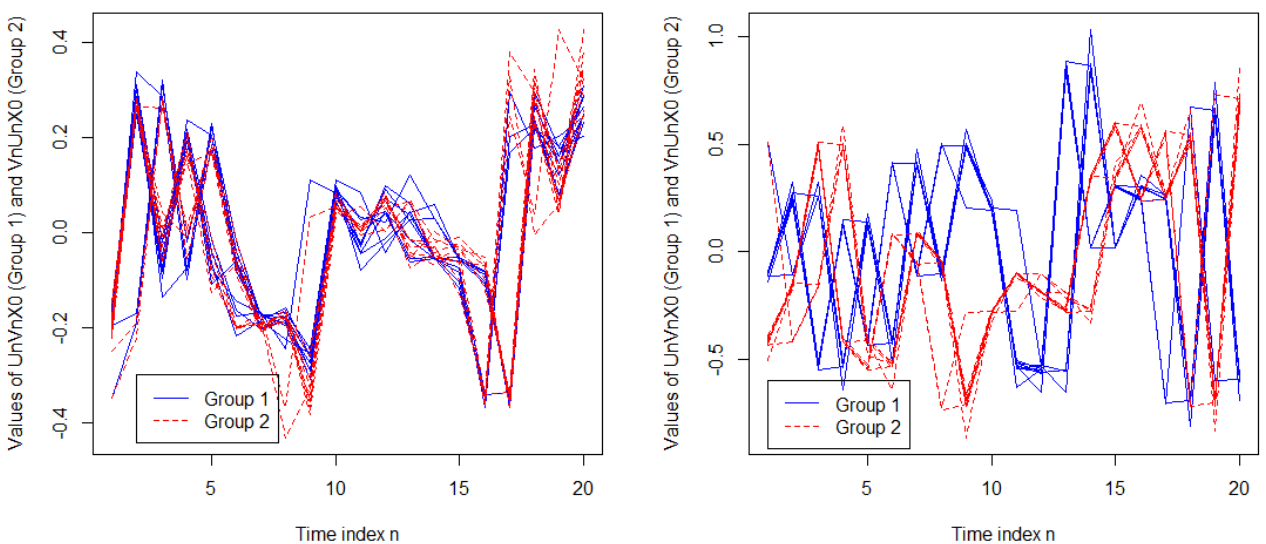

Figure 1: First dimension curves $\left\{\left(U^{n} V^{n}\right) X_{0}, n \in \mathbb{Z}\right\}$ (Group 1) and $\left\{\left(V^{n} U^{n}\right) X_{0}, n \in \mathbb{Z}\right\}$ (Group 2) when $X_{0}$ is close to Im $C_{U, V}$ (left: $\varepsilon_{0}=1$, $\left.\varepsilon_{1}=0.05\right)$ and when $X_{0}$ is far from $\operatorname{Im} C_{U, V}$ (right: $\left.\varepsilon_{0}=0.05, \varepsilon_{1}=1\right)$.

Finally, we illustrate Proposition 24 by a simulation of $\left(X_{n}, n \in \mathbb{Z}\right)$ and a solution $\left(Y_{n}, n \in \mathbb{Z}\right)$ of the form $\left(U^{n} \inf \left(P, C_{P, U}\right) X, n \in \mathbb{Z}\right)$, which is a unique way to write a stationary and stationarily correlated series with $\left(X_{n}, n \in \mathbb{Z}\right)$. Let $\left(v_{1}, \ldots, v_{5}\right)$ be an orthonormal basis of $\mathbb{C}^{5}$. Then let us consider $Q_{1}=v_{1} \otimes v_{1}, Q_{2}=v_{2} \otimes v_{2}+v_{3} \otimes v_{3}$ and $Q_{3}=v_{4} \otimes v_{4}+v_{5} \otimes v_{5}$. We denote by $U$ the unitary operator $U=e^{i \lambda} Q_{1}+e^{i \lambda^{\prime}} Q_{2}+e^{i \lambda^{\prime \prime}} Q_{3}$, which is the shift operator of the series $\left(X_{n}, n \in \mathbb{Z}\right)$ with $X_{n}=U^{n} X_{0}, X_{0}$ being any element of $\mathbb{C}^{5}$.

Let us choose a projector $P=v_{2} \otimes v_{2}+v_{4} \otimes v_{4}$. As $\inf \left(P, Q_{1}\right)=0, \inf \left(P, Q_{2}\right)=v_{2} \otimes v_{2}$ and $\inf \left(P, Q_{3}\right)=v_{4} \otimes v_{4}$, for this projector, the series solution of the form $\left(U^{n} \inf \left(P, C_{P, U}\right) X, n \in \mathbb{Z}\right)$ is $\left(Y_{n}, n \in \mathbb{Z}\right)=\left(e^{i \lambda^{\prime} n} v_{2} \otimes v_{2} X_{0}+e^{i \lambda^{\prime \prime} n} v_{4} \otimes v_{4} X_{0}, n \in \mathbb{Z}\right)$. In Figure 3, the series $\left(Y_{n}, n \in \mathbb{Z}\right)$ appears to have a similar shape as that of the series $\left(X_{n}, n \in \mathbb{Z}\right)$, with less amplitude variations.
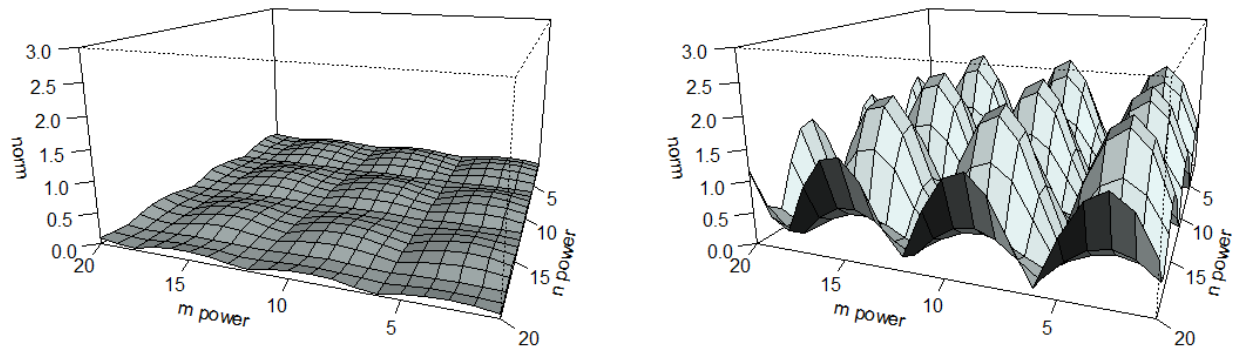

Figure 2: Norms $\left\|U^{n} V^{m} X_{0}-V^{m} U^{n} X_{0}\right\|$ when $X_{0}$ is close to $\operatorname{Im} C_{U, V}\left(\right.$ left: $\left.\varepsilon_{0}=1, \varepsilon_{1}=0.05\right)$ and when $X_{0}$ is far from $\operatorname{Im} C_{U, V}$ (right: $\varepsilon_{0}=0.05$, $\varepsilon_{1}=1$ ), for $n$ and $m$ varying from 1 to 20 . 
A series $\mathrm{Xn}$ and a stationarily correlated solution $\mathrm{Yn}$

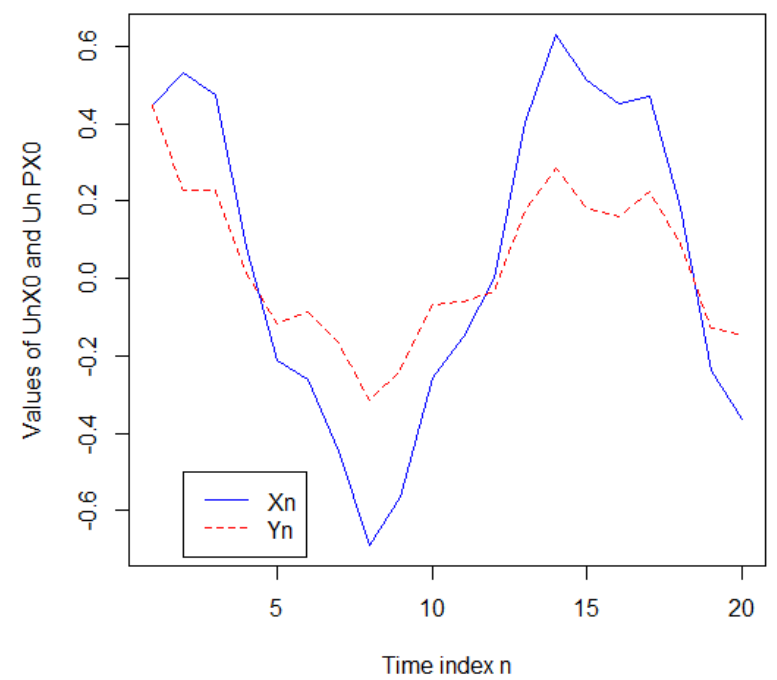

Figure 3: Two stationarily correlated series.

\section{Conclusion}

In closing, we wish to emphasize the similarity between the three types of commuter. The maximal commuters have analogous properties. In particular, $\operatorname{Im} C_{P, D}=\operatorname{Ker}(P D-D P), \operatorname{Im} C_{P, U}=\cap_{n \in \mathbb{Z}} \operatorname{Ker}\left(P U^{n}-U^{n} P\right)$, and $\operatorname{Im} C_{V, U}=$ $\cap_{n, m \in \mathbb{Z}} \operatorname{Ker}\left(V^{m} U^{n}-U^{n} V^{m}\right)$. Note also that $C_{P, U}$ can be written by means of the maximal commuters of projectors, viz. $C_{P, U}=\inf \left\{C_{P, \mathcal{E}_{U} A}: A \in \mathcal{B}_{\Pi}\right\}$, that $C_{V, U}$ can be written by means of a family of maximal commuters $C_{P, U}$ and of a family of commuters of two projectors, i.e., $C_{V, U}=\inf \left\{C_{\mathcal{E}_{V} A, U}: A \in \mathcal{B}_{\Pi}\right\}=\inf \left\{C_{\mathcal{E}_{V} A, \mathcal{E}_{U} B}:(A, B) \in \mathcal{B}_{\Pi} \times \mathcal{B}_{\Pi}\right\}$. In the future, one could consider, for two functional processes, the estimation of common and specific features for their associated shift operators, more precisely, the maximal commuter of the two shift operators.

\section{Acknowledgments}

The authors wish to thank the Editor-in-Chief, Christian Genest, the Editors of the Special Issue, and the reviewers for their constructive remarks which helped them to improve the manuscript significantly.

\section{References}

[1] R. Azencott, D. Dacunha-Castelle, Séries d'observations irrégulières, Masson, Paris, 1984.

[2] M. Benko, A. Kneip, Common functional component modelling, Proceedings of 55th Session of the International Statistical Institute, Sydney, Australia, 2005.

[3] D. Bosq, Linear Processes in Functions Spaces: Theory and Applications, Springer, Berlin, 2000.

[4] D.R. Brillinger, Time Series: Data Analysis and Theory, Holt, Rinehart and Winston, New York, 1975.

[5] A. Boudou, Approximation of the principal components analysis of a stationary function, Statist. Probab. Lett. 76 (2006) 571-578.

[6] A. Boudou, Y. Romain, On spectral and random measures associated to continuous and discrete time processes, Statist. Probab. Lett. 59 (2002) $145-157$.

[7] A. Boudou, Y. Romain, On product measures associated with stationary processes, in: The Oxford Handbook of Functional Data Analysis, Oxford Univ. Press, Oxford, 2011, pp. 423-451.

[8] A. Boudou, S. Viguier-Pla, Une généralisation des fonctions aléatoires stationnairement corrélées, Actes des 45èmes Journées de Statistique, SFdS, Toulouse, Institut Henri-Poincaré, 11, rue Poincaré, Paris, vol. 169 (2013), pp. 1-3.

[9] A. Boudou, S. Viguier-Pla, Gap between orthogonal projectors: Application to stationary processes, J. Multivariate Anal. 46 (2016) $282-300$.

[10] A. Cuevas, A partial overview of the theory of statistics with functional data, J. Statist. Plann. Inf. 147 (2014) 1-23.

[11] D. Dacunha-Castelle, M. Duflo, Probabilités et statistiques, II: Problèmes à temps mobile, Masson, Paris, 1993. 
[12] F. Ferraty, P. Vieu, S. Viguier-Pla, Factor-based comparison of groups of curves, Comp. Stat. Data Anal. 51 (2007) 4903-4910.

[13] L. Horváth, P. Kokoszka, Inference for Functional Data with Application, Springer, New York, 2012.

[14] F. Gamboa, A. Rouault, Operator-values spectral measures and large deviation, J. Statist. Plann. Inf. 154 (2014) $72-86$.

[15] T. Hsing, R. Eubank, Theoretical Foundations of Functional Data Analysis, with an Introduction to Linear Operators, Wiley, New York, 2015.

[16] N.J. Laustsen, Commutators of operators on Banach spaces, J. Operator Theory 48 (2002) 503-514.

[17] G. Lindgren, Stationary Stochastic Processes: Theory and Applications, Taylor \& Francis, New York, 2013.

[18] J.O. Ramsay, B.W. Silverman, Applied Functional Data Analysis: Methods and Case Studies, Springer, New York, 2002.

[19] F. Riesz, B. Nagy, Functional Analysis, Dover Publications, New York, 1991. 